DOI https://doi.org/10.24297/jam.v20i.9044

\title{
On the existence of positive solutions for a nonlinear elliptic class of equations in $\mathbb{R}^{2}$ and $\mathbb{R}^{3}$
}

\author{
José R. Quintero
}

Mathematics Department, Science Faculty, Universidad del Valle, Colombia

jose.quintero@correounivalle.edu.co

\begin{abstract}
We study the existence of positive solutions for an elliptic equation in $\mathbb{R}^{N}$ for $N=2,3$ which is related with the existence of standing (localized) waves and the existence of the ground state solutions for some physical model or systems in fluid mechanics to describe the evolution of weakly nonlinear water waves. We use a variational approach and the well-known principle of concentration-compactness due to P. Lions to obtain the existence of this type of solutions, even in the case that the nonlinear term $g$ is a non-homogeneous function or an operator defined in $H^{1}\left(\mathbb{R}^{N}\right)$ with values in $\mathbb{R}$.
\end{abstract}

Keywords: Elliptic equation, concentration-compactness, variational approach.

Mathematics Subject Classifications: 35Q55; 35J20; 35B09.

\section{Introduction}

The first step in the study of some interesting physical problems in acoustics, in the context of gravity waves, in fluid mechanics, or optics is to establish the existence of special positive solutions as travelling and standing waves, as happens in the case of the generalized Schrödinger equation, the generalized Davey-Stewartson type systems, the Zakharov-Rubenchik (also known Benney-Roskes) system and the generalization the Zakharov-Rubenchik system. The main issue in previous models is that the existence of such special solutions is reduced to studying the existence of solutions for a single Schrödinger type nonlinear equation of the form

$$
i \partial_{t} \psi+\sigma_{1} \Delta_{\perp} \psi+\epsilon \partial_{z}^{2} \psi=\sigma|\psi|^{p} \psi-\beta|\psi|^{2} \psi-\gamma E_{0}\left(|\psi|^{2}\right) \psi,
$$

where $\sigma_{1}, \epsilon, \sigma, \beta, \gamma \in \mathbb{R}, E_{0}$ is a nonlocal linear operator defined via a Fourier multiplier, $\mathbf{x} \in \mathbb{R}^{N}$ for $N=2,3$, and $\Delta=\Delta_{\perp}+\partial_{z}^{2}$ with $\Delta_{\perp}=\partial_{x}^{2}$ in the case $N=2$. In this general case, if we look for standing wave solutions for (1) of the form $\psi(\mathbf{x}, t)=e^{i c t} u(\mathbf{x})$, then $u$ satisfies the equation

$$
-c u+\sigma_{1} \Delta_{\perp} u+\epsilon \partial_{z}^{2} u=\sigma|u|^{p} u-\beta|u|^{2} u-\gamma E_{0}\left(|u|^{2}\right) u,
$$

something that also happens in the case of generalization of the KdV equation or the Gardner equation in $\mathbb{R}^{N}$ for $\gamma=0$. We note that this equation is elliptic in the case $\epsilon \sigma_{1}>0$.

Regarding the existence or non existence of positive solutions for nonlinear models, there are plenty of result for different kind of problems in the elliptic and non elliptic case. For instance, J. Gidaglia and J.C Saut in [7] showed a non existence result of non trivial solutions for the nonlinear equation

$$
-\omega v+\sum_{j=1}^{N} \epsilon_{j} \partial_{j}^{2} v+f(|v|) v=0
$$


in the case $\epsilon_{j}= \pm 1$ with $\epsilon_{k} \neq \epsilon_{l}$ for some $(k, l)$, under the assumption that the nonsingular diagonal matrix $\operatorname{diag}\left(e_{1}, \cdots, e_{N}\right)$ is not positive-definite (resp. negative definite) and that $f$ is a continuous real-valued function such that

$$
|f(s)| \leq C\left(1+|s|^{p}\right), \quad p \leq \frac{2}{N-2}, \quad N \neq 2, \quad 1 \leq p<\infty, \quad N=2 .
$$

Moreover, the existence of a positive (radial) solution $g \in C^{\infty}\left(\mathbb{R}^{2}\right) \cap H^{1}\left(\mathbb{R}^{2}\right)$ of the elliptic equation

$$
-g+\Delta g+g^{3}=0
$$

implies the existence of a nontrivial solutions of the nonelliptic equation

$$
-w+w_{x x}-w_{y y}+w^{3}=0
$$

Moreover, if we set $u(x, y, t)=e^{i t} w(x, y)$, then $u$ satisfies the equation

$$
i u_{t}+u_{x x}-u_{y y}+|u|^{2} u=0 .
$$

On the other hand, for $p \in\left(1, \frac{N+2}{N-2}\right)$ with $N>2$ and $b \in C\left(\mathbb{R}^{N}\right)$ such that $b(x) \rightarrow b_{\infty}$ as $|x| \rightarrow \infty$, J. Lions $[9]$ established the solvability of the elliptic equation

$$
-\Delta u+u=b(x) u^{p}=0
$$

This result was extended by Bahri and Lions [2] to the case when $b(x) \geq b_{\infty}-C e^{-\delta|x|}$. In the case that $b(x)<b_{\infty}$ and $b^{Y}$ is a convex combination of functions $b(\cdot-y)$ with $y=\left(z_{1}, \cdots, z_{N}\right)$ and $z_{i} \in \mathbb{Z}$, then K. Tintarev in $[15]$ showed the existence of a nontrivial solution of the elliptic equation

$$
-\Delta u+u=b^{Y}(x) u^{p}=0
$$

In this paper we are interested in establishing a general existence result of positive solutions of the special elliptic equation in $\mathbb{R}^{N}(N=2,3)$

$$
c u-\Delta u+g(u)=0, \quad c>0
$$

where $g$ is either a function defined in $\mathbb{R}$ or an operator defined in $H^{1}\left(\mathbb{R}^{N}\right)$ having some variational properties. In particular, we are very interested in this elliptic equation due to its relation with the existence of special solutions for some well known systems. For instance, we consider the existence of standing (localized) waves $v(\mathbf{x}, t)=e^{i c t} u(\mathbf{x})$ for the second order differential equation in $\mathbb{R}^{N}$

$$
i u_{t}+\Delta u-g(u)=0
$$

where $g$ is a function or an operator defined such that $g(u)=\tilde{g}(|u|) u$. In this case, we find that $u$ satisfies the elliptic equation (2). We note that equation (3) is related with the generalized Schrödinger equation in the case

$$
g(u)=a|u|^{p} u
$$

with the Davey-Stewartson type systems in the case

$$
g(u)=a|u|^{p} u+b E_{1}\left(|u|^{2}\right) u
$$

with the Benney-Roskes/Zakharov-Rubenchik system in the case

$$
g(u)=a|u|^{2} u+b E_{2}\left(|u|^{2}\right) u
$$


and with the generalization of the Zakharov-Rubenchik system in the case

$$
g(u)=a|u|^{p} u+b|u|^{2} u+c E_{3}\left(|u|^{2}\right) u,
$$

where $E_{j}(j=1,2,3)$ is a linear operator defined on $H^{1}\left(\mathbb{R}^{N}\right)$ via a Fourier multiplier of the form

$$
\widehat{E}_{j}(u)(\xi)=\Gamma_{j}(\xi) \widehat{u}(\xi)
$$

We refer to the following works to get more details for these models: [4], [5], [6], [11], [12], [13].

On the other hand, if we consider the existence of ground state solutions $(x$-travelling $) v(\mathbf{x}, t)=u(x-c t, z)(\mathbf{x}=(x, z))$ for the second order differential equation in $\mathbb{R}^{N}$

$$
u_{t}+\Delta u_{x}-(g(u))_{x}=0
$$

we see that $u$ satisfies the elliptic equation (2). In this case, the nonlinear model (4) with nonlinearity $g(s)=a s^{p}+b s^{q}$ could be though as a generalization of the $\mathrm{KdV}$ equation in $\mathbb{R}^{N}(a=0, q=2)$, a generalization of the modified $\mathrm{KdV}$ equation in $\mathbb{R}^{N}(b=0, p=3)$, a generalization of the quadratic-cubic $\mathrm{KdV}$ equation in $\mathbb{R}^{N}(a \neq 0, b \neq 0, p=3, q=2)$, and a generalization of the Gardner equation in $\mathbb{R}^{N}(a \neq 0, b \neq 0, p=2 q)$.

The existence of solutions for the nonlinear elliptic model 2 is a consequence of the variational characterization of solutions and the well-known concentration-compactness principle by P. Lions, although the non-linear term $g$ is not necessarily an homogeneous function or operator. The result is inspired in Cipolatti's approach in [4] related with the existence of standing waves for a Davey-Stewartson system.

Remark 1.1 The positiveness of solutions for the elliptic equation (2) in Theorem (3.1) and Theorem (3.2) bellow follows from the work by T. Cazenave establish in [3], page 168.

Lemma 1.1 Let $a: \mathbb{R}^{N} \rightarrow \mathbb{R}$ be a continuous function and let us assume that $a(x) \rightarrow 0$, as $|x| \rightarrow \infty$. Let us assume further that there exists $v \in H^{1}\left(\mathbb{R}^{N}\right)$ such that

$$
J(v)=\int_{\mathbb{R}^{N}}\left(|\nabla v|^{2}-a(\mathbf{x}) v^{2}\right) d \mathbf{x}<0
$$

Then, there exists $\lambda>0$ and a positive solution $u \in H^{1}\left(\mathbb{R}^{N}\right)$ of the equation

$$
-\Delta u+\lambda u=a(\mathbf{x}) u \text {. }
$$

In addition, if $w \in H^{1}\left(\mathbb{R}^{N}\right)$ is nonnegative, $w \neq 0$, is such that

$$
-\Delta w+\nu w=a(\mathbf{x}) w
$$

for some $\nu \in \mathbb{R}$, then $w=\rho u$ for some $\rho>0$. In particular, $\nu=\lambda$.

This paper is organized as follows. In section 2, we include some preliminary results, state the main hypotheses on the operator $g$ and provide a variational characterization of the ground state solutions for the general equation (3) and (4). In section 3, we prove the main results by using the variational characterization of ground state solutions and the Concentration-Compactness principle by P. Lions ([9, 10]). In section 4, we provide some non trivial examples, which include generalizations of $\mathrm{KdV}$ type model in $\mathbb{R}^{N}$, Davey-Stewartson type systems (see [4]), Benney-Roskes/Zakharov-Rubenchik system in spatial dimensions $N=2,3$ (see [1], [5], [11], [14]), the generalized Benney-Roskes/Zakharov-Rubenchik system in spatial dimensions $N=2,3$ ([12], [13]). As far as our knowledge goes, the last result is new to the literature. 


\section{Preliminaries}

We assume that the nonlinear term $g$ is such that the model has a Hamiltonian structure. In other words, we assume that there is an operator $\Xi$ defined in $H^{1}\left(\mathbb{R}^{N}\right)$ such that $\Xi^{\prime}(\phi)=g(\phi)$ for $\phi \in H^{1}\left(\mathbb{R}^{N}\right)$. From previous assumption, we see directly that standing waves of the form $v(\mathbf{x}, t)=e^{i c t} u(\mathbf{x})$ for 3 for $g(u)=\tilde{g}(|u|) u$ and travelling wave solutions in the $x$-direction of the form $v(\mathbf{x}, t)=u(x-c t, z)(\mathbf{x}=x, z))$ for 4 ) are characterized as critical points of the functional

$$
\mathcal{F}(u)=\mathcal{H}(u)+c Q(u)
$$

where the Hamiltonian $\mathcal{H}$ and the charge $Q$ are defined respectively by

$$
\mathcal{H}(u)=\frac{1}{2} \int_{R^{N}}|\nabla u|^{2} d \mathbf{x}+\Xi(u), \quad Q(u)=\frac{1}{2} \int_{R^{N}} u^{2} d \mathbf{x} .
$$

Moreover, we have that $\mathcal{F}^{\prime}(u)(v)=0$ for any $v \in H^{1}\left(\mathbb{R}^{N}\right)$ is equivalent to have solutions for the equation

$$
c u-\Delta u+g(u)=0, \quad \text { in } \mathbb{R}^{N}
$$

If we set the functionals $T$ and $V$ on $H^{1}\left(\mathbb{R}^{N}\right)$ as

$$
T(u)=\|\nabla u\|_{2}^{2}, \quad V(u)=\frac{c}{2} \int_{\mathbb{R}^{N}} u^{2} d \mathbf{x}+\Xi(u)
$$

we see that

$$
\mathcal{H}(u)=\mathcal{F}(u)-\frac{c}{2}\|u\|_{2}^{2}
$$

Before we go further, we note that any nontrivial solution $u$ of the equation (2) satisfies the identities

$$
V(u)=-\frac{(N-2)}{2 N} T(u), \quad \mathcal{F}(u)=\frac{1}{N} T(u)
$$

which imply that $V(u)=0$ for $N=2$ and $V(u)<0$ for $N=3$. These facts will be clever in the minimization argument for $N=2$ and $N=3$, as in the work by R. Cipolatti in [4].

Now, for each $\mu \in \mathbb{R}$ we define the level set for $V$

$$
\Sigma_{\mu}=\left\{\psi \in H^{1}\left(\mathbb{R}^{N}\right) \backslash\{0\}: V(\psi)=\mu\right\}
$$

and the infimum $j(\mu)$ by

$$
j(\mu)=\inf \left\{\frac{1}{2} T(\psi): \psi \in \Sigma_{\mu}\right\} .
$$

As we mention above, we need to impose some natural conditions on the functional $\Xi$. Hereafter, we assume that

$$
\Xi(u)=\int_{\mathbb{R}^{N}} G(u) d \mathbf{x} .
$$

\section{Conditions on $G$}

(G1) For $N=2,3$, there are $0<r_{j}<\frac{4}{N-2}$ with $1 \leq j \leq k$ and $M>0$ such that for $\phi \in H^{1}\left(\mathbb{R}^{N}\right)$

$$
\int_{\mathbb{R}^{N}}|G(\phi)| d \mathbf{x} \leq M \sum_{j=1}^{k}\|\phi\|_{r_{j}+2}^{r_{j}+2}
$$

(G2) There is $\varphi_{0} \in H^{1}\left(\mathbb{R}^{N}\right)$ such that $V\left(\varphi_{0}\right)<0$. 
(G3) There is $m>2$ such that for $\lambda>0$ and $\psi \in H^{1}\left(\mathbb{R}^{N}\right)$, we have that $G(\lambda \psi)=\lambda^{m} F(\lambda, \psi)$, where $F$ is a continuous operator such that for $u \in H^{1}\left(\mathbb{R}^{N}\right) \backslash\{0\}$

$$
\lim _{\lambda \rightarrow 0}\left|\int_{\mathbb{R}^{N}} F(\lambda, u) d \mathbf{x}\right|<\infty .
$$

(G4) For $\psi \in H^{1}\left(\mathbb{R}^{2}\right) \backslash\{0\}$ such that $V(\psi)=0$, we have that $h_{\psi}(\lambda)<0$, for $\lambda>1$, but close to $1^{+}$.

From condition (G3), for a given $\psi \in H^{1}\left(\mathbb{R}^{N}\right)$ we have that

$$
h_{\psi}(\lambda)=V(\lambda \psi)=\lambda^{2}\left(\frac{c}{2}\|\psi\|_{2}^{2}+\lambda^{m-2} \int_{\mathbb{R}^{N}} F(\lambda, \psi) d \mathbf{x}\right) .
$$

Remark 2.1 On the condition (G4). We want to point out that the condition (3.9) in R. Cipolatti's work [4] is trivially achieved in the case $\alpha<2$, but it unclear in the case $\alpha \geq 2$. It seems that the condition must be that $\left.\frac{d}{d \lambda} V_{1}(\lambda \psi)\right|_{\lambda=1}>0$ for $\psi \in \Sigma_{0}$, where

$$
V_{1}(\psi)=-\frac{\omega}{2}\|\psi\|_{2}^{2}-\frac{a}{\alpha+2}\|\psi\|_{\alpha+2}^{\alpha+2}+\frac{b}{4} B_{1}\left(|\psi|^{2}\right)
$$

with $B_{1}$ being defined as

$$
B_{1}(\psi)=\int_{\mathbb{R}^{N}} E_{1}(\psi) \bar{\psi} d \mathbf{x}
$$

In order to assure that the condition (3.9) in Cipolatti's work holds, we introduce the condition (G4), which generates some simple restrictions on the set $\mathcal{R}_{\omega, b}$ in Cipolatti's work.

Under those conditions on $G$ we are able to establish the following result.

Lemma 2.1 (i) $\Sigma_{\mu} \neq \emptyset$ for any $\mu \in \mathbb{R}$.

(ii) Let $N=2$ and assume conditions $(\mathbf{G} \mathbf{1})-(\mathbf{G} 4)$, then there is $I>0$ such that $j(\mu)=I$ for $\mu \in \mathbb{R}$.

(iii) Let $N=3$ and assume conditions (G1)-(G3), then there is $I>0$ such that $j(\mu)=-\mu^{\frac{1}{3}} I$ for $\mu<0$.

Proof. (i) Let $\psi \in H^{1}\left(\mathbb{R}^{N}\right)$ with $V(\psi) \neq 0$. Then for any $\lambda>0$, we define $\psi_{\lambda}(x)=\psi(y)$, where $x=\sqrt[N]{\lambda} y$. We see directly from the definition of $V$ that

$$
V\left(\psi_{\lambda}\right)=\lambda V(\psi)
$$

On the other hand, from (6)

$$
h_{\psi}(\lambda)=\lambda^{2}\left(\frac{c}{2}\|\psi\|_{2}^{2}+\lambda^{m-2} \int_{\mathbb{R}^{N}} F(\lambda, \psi)(x z) d \mathbf{x}\right)=\lambda^{2} \tilde{h}_{\psi}(\lambda) .
$$

From this fact, we have that $\tilde{h}_{\psi}(\epsilon)>0$, and that $h_{\psi}(\epsilon)=V(\epsilon \psi)>0$, for $\epsilon>0$ small enough. We choose $\lambda>0$ appropriate such that $\lambda V(\epsilon \psi)=\mu>0$, which means that $\Sigma_{\mu} \neq \emptyset$ for any $\mu>0$, since $V\left((\epsilon \psi)_{\lambda}\right)=\lambda V(\epsilon \psi)=\mu$. On the other hand, using condition (G2), there is $\varphi_{0} \in H^{1}\left(\mathbb{R}^{N}\right)$ such that $V\left(\varphi_{0}\right)<0$. So, from condition (G3) there $0<\gamma_{0}<1$ such that $\tilde{h}_{\varphi_{0}}\left(\gamma_{0}\right)=0$, since $\tilde{h}_{\varphi_{0}}(0)>0$ and $\tilde{h}_{\varphi_{0}}(1)<0$. In other words, we have that $\Sigma_{0} \neq \emptyset$. Now, for $\mu<0$, we choose $\lambda>0$ large enough such that $V\left(\left(\varphi_{0}\right)_{\lambda}\right)=\lambda V\left(\varphi_{0}\right)=\mu<0$, which means that $\Sigma_{\mu} \neq \emptyset$ for any $\mu<0$.

Now we are going to prove (ii). Let $N=2$ and set

$$
I=j(0)=\inf \left\{\frac{1}{2} T(\psi): \psi \in \Sigma_{0}\right\} .
$$


We claim that $I>0$. To see this we need to recall the Gagliardo-Nirenberg-Sobolev inequality in $\mathbb{R}^{m}$ for $m \geq 2$ and $0<r<\frac{4}{m-2}$

$$
\|f\|_{r+2}^{r+2} \leq C(r, m)\|\nabla f\|_{2}^{\frac{r m}{2}}\|f\|_{2}^{2+\frac{r}{2}(2-m)} .
$$

From the Gagliardo-Nirenberg-Sobolev inequality for $N=m=2$, the condition (G1) with $0<r_{j}<\infty$ for $1 \leq j \leq k$, and that $V(\psi)=0$, we conclude that

$$
\frac{c}{2}\|\psi\|_{L^{2}\left(\mathbb{R}^{2}\right)}^{2}=\left|\int_{\mathbb{R}^{2}}\right| G(\phi)(z)|d \mathbf{x}| \leq M \sum_{j=1}^{k}\|\phi\|_{r_{j}+2}^{r_{j}+2} \leq M_{1}\left(\sum_{j=1}^{k}\|\nabla \psi\|_{L^{2}\left(\mathbb{R}^{2}\right)}^{r_{j}}\right)\|\psi\|_{L^{2}\left(\mathbb{R}^{2}\right)}^{2},
$$

which implies that $I>0$. On the other hand, for $\lambda>0$ we have that

$$
\psi \in \Sigma_{\mu} \quad \Leftrightarrow \quad \psi_{\lambda} \in \Sigma_{\mu \lambda}
$$

If we set for $\lambda>0, \varphi_{\lambda}(y)=\varphi(x)$ with $x=\sqrt{\lambda} y$, we have that $T\left(\varphi_{\lambda}\right)=T(\varphi)$. From this we have necessarily that the function $j(\mu)$ is constant on $(-\infty, 0)$ and $(0, \infty)$. We will see that $j(\mu)=I$ for any $\mu \in \mathbb{R}$. From the definition of $I$, given $\epsilon>0$ there is $\varphi \in \Sigma_{0}$ such that

$$
I<\frac{1}{2} T(\varphi)<I+\epsilon .
$$

Now, take a sequence $\left(\mu_{n}\right)_{n}$ such that $\mu_{n} \rightarrow 0^{-}$. From the fact that $h_{\varphi}(0)=h_{\varphi}(1)=0$, and the condition (G4), we see that there is $\alpha_{n}>1$ such that $h_{\varphi}\left(\alpha_{n}\right)=\mu_{n}$ and $\alpha_{n} \rightarrow 1^{+}$, which implies that

$$
\frac{1}{2} T\left(\alpha_{n} \varphi\right)=\frac{\alpha_{n}^{2}}{2} T(\varphi) \geq j\left(\mu_{n}\right) .
$$

Then, we also have that

$$
j\left(\mu_{n}\right)-I<\frac{1}{2}\left(\alpha_{n}^{2}-1\right) T(\varphi)+\epsilon
$$

which implies that

$$
\limsup _{n \rightarrow \infty} j\left(\mu_{n}\right) \leq I \text {. }
$$

Now, from the definition of $j\left(\mu_{n}\right)$, given $\epsilon>0$, there is $\varphi_{n} \in \Sigma_{\mu_{n}}$ such that

$$
j\left(\mu_{n}\right)<\frac{1}{2} T\left(\varphi_{n}\right)<j\left(\mu_{n}\right)+\epsilon .
$$

From the fact that $\tilde{h}_{\varphi_{n}}(0)>0$ and $\tilde{h}_{\varphi_{n}}(1)=\mu_{n}<0$, there is $0<\alpha_{n}<1$ such that $h_{\varphi_{n}}\left(\alpha_{n}\right)=\alpha_{n}^{2} \tilde{h}_{\varphi_{n}}\left(\alpha_{n}\right)=0$. So, we conclude that

which implies that

$$
I \leq \frac{1}{2} T\left(\alpha_{n} \varphi_{n}\right)=\frac{1}{2} \alpha_{n}^{2} T\left(\varphi_{n}\right)<\frac{1}{2} T\left(\varphi_{n}\right)<j\left(\mu_{n}\right)+\epsilon
$$

$$
\liminf _{n \rightarrow \infty} j\left(\mu_{n}\right) \geq I \text {. }
$$

So, we have established that $j(\mu)=I$ for $\mu \leq 0$. An analogous argument shows that $j(\mu)=I$ for $\mu \geq 0$, as desired.

Now we proceed to establish (iii) for $N=3$. We set

$$
I=j(-1)=\inf \left\{\frac{1}{2} T(\psi): \psi \in \Sigma_{-1}\right\} .
$$

As in previous case, from the Gagliardo-Nirenberg-Sobolev inequality, the condition (G1) with $0<r_{j}<4$ for $1 \leq j \leq k$, and that $V(\psi)=-1$, we conclude that $I>0$. In fact, assume that $I=0$. So, choose a sequence $\left(\psi_{n}\right)_{n} \subset H^{1}\left(\mathbb{R}^{3}\right)$ such that $V\left(\psi_{n}\right)=-1$ and $\left\|\nabla \psi_{n}\right\|_{2} \rightarrow 0$, as $n \rightarrow \infty$. If for some subsequence $\left(\psi_{n_{k}}\right)_{k} \subset\left(\psi_{n}\right)_{n}$ we have that $\left\|\psi_{n_{k}}\right\|_{2} \rightarrow 0$, as $k \rightarrow \infty$, then we have from the Gagliardo-Nirenberg-Sobolev inequality that $\left\|\psi_{n_{k}}\right\|_{r_{j}+2} \rightarrow 0$, as $k \rightarrow \infty$, since

$$
\left\|\psi_{n_{k}}\right\|_{r_{j}+2}^{r_{j}+2} \leq C\left(r_{j}\right)\left\|\nabla \psi_{n_{k}}\right\|_{2}^{\frac{3 r_{j}}{2}}\left\|\psi_{n_{k}}\right\|_{2}^{2-\frac{r_{j}}{2}}
$$


So, we reach the contradiction

$$
-1=\lim _{k \rightarrow \infty} V\left(\psi_{n_{k}}\right)=0
$$

In other words, $\left\|\psi_{n}\right\|_{2} \geq L$ for some $L>0$ and $n \in \mathbb{N}$. From this and the Gagliardo-Nirenberg-Sobolev inequality, we conclude for some $0<r_{j}<2$ that

$$
\lim _{n \rightarrow \infty}\left(\frac{\left|\int_{\mathbb{R}^{3}} G\left(\psi_{n}\right) d \mathbf{x}\right|}{\left\|\psi_{n}\right\|_{2}}\right) \leq \lim _{n \rightarrow \infty} M \sum_{j=1}^{k}\left(\frac{\left\|\nabla \psi_{n}\right\|_{2}^{\frac{3 r_{j}}{2}}}{\left\|\psi_{n}\right\|_{2}^{\frac{r_{j}}{2}}}\right)=0
$$

implying that

$$
-\lim _{n \rightarrow \infty}\left(\frac{1}{\left\|\psi_{n}\right\|_{2}}\right)=\frac{c}{2}+\lim _{n \rightarrow \infty}\left(\frac{\int_{\mathbb{R}^{3}} G\left(\psi_{n}\right) d \mathbf{x}}{\left\|\psi_{n}\right\|_{2}}\right)=\frac{c}{2},
$$

which again is a contradiction. So, we have that $I>0$. On the other hand, for $\lambda>0$ we have that

$$
\psi \in \Sigma_{\mu} \quad \Leftrightarrow \quad \psi_{\lambda} \in \Sigma_{\mu \lambda}
$$

If we set for $\lambda>0, \varphi_{\lambda}(y)=\varphi(x)$ with $x=\lambda^{\frac{1}{3}} y$, we have that $T\left(\varphi_{\lambda}\right)=\lambda^{\frac{1}{3}} T(\varphi)$. From this, we conclude that $j(\mu)=-\mu^{\frac{1}{3}} I$ for $\mu<0$.

We see now that the minimization problem associated with $j$ has an equivalent formulation.

Theorem 2.2 For $N=2$, the minimization problem

$$
\left\{\begin{aligned}
\varphi & \in \Sigma_{0}, \quad \varphi \neq 0 \\
T(\varphi) & =\inf \{T(\psi): V(\psi)=0\}
\end{aligned}\right.
$$

is equivalent to the minimization problem

$$
\left\{\begin{aligned}
\varphi & \in H^{1}\left(\mathbb{R}^{2}\right), \quad \varphi \neq 0 \\
T(\varphi) & =\inf \{T(\psi): V(\psi) \leq 0\}
\end{aligned}\right.
$$

Proof. Let

$$
\tilde{I}_{0}=\inf \{T(\psi): V(\psi) \leq 0\}, \quad I_{0}=\inf \{T(\psi): V(\psi)=0\} .
$$

Clearly, we have that $\tilde{I}_{0} \leq I_{0}$. Let $\psi \in H^{1}$ be such that $\psi \neq 0$ and $V(\psi)<0$. We set the function $h_{\psi}(\lambda)=V(\lambda \psi)$. We know that $h_{\psi}(1)<0$ and that $h_{\psi}(0)=0$, so there is $0<\lambda<1$ such that $h_{\psi}(\lambda)=V(\lambda \psi)=0$. Then, we conclude that

$$
I_{0} \leq T(\lambda \psi)=\lambda^{2} T(\psi) \leq T(\psi)
$$

meaning that $I_{0} \leq \tilde{I}_{0}$.

Following the same arguments, we also have that

Theorem 2.3 For $N=3$ and $\mu<0$, the minimization problem

$$
\left\{\begin{aligned}
\varphi & \in \Sigma_{\mu}, \quad \varphi \neq 0 \\
T(\varphi) & =\inf \{T(\psi): V(\psi)=\mu\}
\end{aligned}\right.
$$

is equivalent to the minimization problem

$$
\left\{\begin{array}{l}
V(\varphi)=\mu, \quad \varphi \neq 0 \\
T(\varphi)=\inf \{T(\psi): V(\psi) \leq \mu\}
\end{array}\right.
$$




\section{Main Results}

We split the proof in the cases $N=2$ and $N=3$. In the case $N=2$, we use conditions (G1)-(G4), that the embedding $H^{1}(U) \subset L^{q}(U)$ is compact, for $1 \leq q<\infty$ and $U \subset \mathbb{R}^{2}$ bounded, the concentration-compactness and the variational characterization $j$.

Theorem 3.1 (i) $X=\left\{\psi \in H^{1}\left(\mathbb{R}^{2}\right): \psi \neq 0\right.$, $\psi$ solves $\left.(2)\right\}$ has a function.

(ii) $\varphi \in X$ if and only if $\varphi$ solves the minimization problem

$$
\left\{\begin{aligned}
\varphi & \in \Sigma_{0} \\
T(\varphi) & =\inf \{T(\psi): V(\psi)=0\}
\end{aligned}\right.
$$

Proof. Let $j(\mu)$ be defined in $(5)$. We want to show that the problem 12 has a solution. In fact, let $\left(\varphi_{n}\right)_{n} \subset \Sigma_{0}$ such that

$$
I_{0}=\inf \{T(\psi): V(\psi)=0\}=\lim _{n \rightarrow \infty} T\left(\varphi_{n}\right) .
$$

Set $\alpha_{n}=-\left\|\varphi_{n}\right\|_{2}^{2}$ and define the function $\phi_{n}(x)=\varphi_{n}(y)$ with $y=\sqrt{-\alpha_{n}} x$. Then we have that $T\left(\phi_{n}\right)=T\left(\varphi_{n}\right)$ and $-\alpha_{n} V\left(\phi_{n}\right)=V\left(\varphi_{n}\right)=0$, meaning that $\left(\phi_{n}\right)_{n} \subset \Sigma_{0}$ is a minimizing sequence with

$$
\left\|\phi_{n}\right\|_{2}^{2}=1
$$

Moreover, due to the fact that $\left(\phi_{n}\right)_{n} \subset \Sigma_{0}$ is a minimizing sequence for $I_{0}$, we have that $\left\|\nabla \phi_{n}\right\|_{2}$ is also a bounded sequence in $L^{2}\left(\mathbb{R}^{2}\right)$. So, there is $\phi_{0} \in H^{1}\left(\mathbb{R}^{2}\right)$ such that $\phi_{n} \rightarrow \phi_{0}$ (weakly) in $H^{1}\left(\mathbb{R}^{2}\right)$.

Now, we set the measure $\nu_{n}$ with density $\rho\left(\phi_{n}\right)$ with respect to the Lebesgue measure given by

$$
\rho(\phi)=|\nabla \phi|^{2}+|\phi|^{2}
$$

So, we have that

$$
\int_{\mathbb{R}^{2}} d \nu_{n}=\int_{\mathbb{R}^{2}} \rho\left(\phi_{n}\right) d \mathbf{x}:=\sigma_{n} \rightarrow \sigma_{0}=I_{0}+1, \quad n \rightarrow \infty .
$$

We now apply the Lions' Concentration-Compactness Principle (see [9]-[10]). First, we see that vanishing is not possible. In fact, from the Sobolev inequality we have for any open box $J$ in $\mathbb{R}^{2}$ and for any $r_{j}>0$ that

$$
\int_{J}\left|\phi_{n}\right|^{r_{j}+2} d \mathbf{x} \leq C\left(\int_{J}\left(\left|\nabla \phi_{n}\right|^{2}+\left|\phi_{n}\right|^{2}\right) d \mathbf{x}\right)^{\frac{r_{j}+2}{2}} .
$$

Now, covering $\mathbb{R}^{2}$ with a sequence of open boxes $J_{k}$ in such a way that $J_{k} \cap J_{m}=\emptyset$, then we see that

$$
\begin{aligned}
\left\|\phi_{n}\right\|_{r_{j}+2}^{r_{j}+2} & =\sum_{k} \int_{J_{k}}\left|\phi_{n}\right|^{r_{j}+2} d \mathbf{x} \leq C \sum_{k}\left(\int_{J_{k}}\left(\left|\nabla \phi_{n}\right|^{2}+\left|\phi_{n}\right|^{2}\right) d \mathbf{x}\right)^{\frac{r_{j}+2}{2}} \\
& \leq C \sum_{k}\left(\int_{J_{k}}\left(\left|\nabla \phi_{n}\right|^{2}+\left|\phi_{n}\right|^{2}\right) d \mathbf{x}\right)^{\frac{r_{j}}{2}} \int_{J_{k}}\left(\left|\nabla \phi_{n}\right|^{2}+\left|\phi_{n}\right|^{2}\right) d \mathbf{x} \\
& \leq C\left(\sup _{k} \int_{J_{k}}\left(\left|\nabla \phi_{n}\right|^{2}+\left|\phi_{n}\right|^{2}\right) d \mathbf{x}\right)^{\frac{r_{j}}{2}} \sum_{k} \int_{J_{k}}\left(\left|\nabla \phi_{n}\right|^{2}+\left|\phi_{n}\right|^{2}\right) d \mathbf{x} \\
& \leq\left. C\left(\sup _{k} \int_{J_{k}}\left(\left|\nabla \phi_{n}\right|^{2}+\left|\phi_{n}\right|^{2}\right) d \mathbf{x}\right)^{\frac{r_{j}}{2}}|| \phi_{n}\right|_{H^{1}\left(\mathbb{R}^{2}\right)} ^{2} .
\end{aligned}
$$

On the other hand, from $V\left(\phi_{n}\right)=0$ and condition (G1), we conclude that

$$
\frac{c}{2}=\frac{c}{2}\left\|\left.\phi_{n}\right|_{2} ^{2} \leq M \sum_{j=1}^{k}\right\| \phi_{n} \|_{r_{j}+2}^{r_{j}+2}
$$


which gives us a contradiction, since $\left\|\phi_{n}\right\|_{r_{j}+2} \rightarrow 0$, as $n \rightarrow \infty$ for $r_{j}>0$, in the case that we had vanishing.

If we assume Dichotomy, then there is $0<\gamma<\sigma_{0}$ such that for a given $\epsilon>0$, there exist $R_{0}>0$, a sequence $\left(y_{n}\right)_{n} \subset \mathbb{R}^{2}$, $R_{n} \uparrow+\infty$, and a bounded sequence $\left(\phi_{n}^{i}\right)_{n} \subset H^{1}\left(\mathbb{R}^{2}\right)$ for $i=1,2$ (all depending on $\epsilon$ ) such that

$$
\begin{aligned}
& \operatorname{supp}\left(\phi_{n}^{1}\right) \subset B_{R_{0}}\left(x_{n}\right), \operatorname{supp}\left(\phi_{n}^{2}\right) \subset \mathbb{R}^{2} \backslash B_{R_{n}}\left(x_{n}\right) \\
& \left\|\phi_{n}-\phi_{n}^{1}-\phi_{n}^{2}\right\|_{H^{1}\left(\mathbb{R}^{2}\right)} \leq \epsilon \\
& \left|\left\|\nabla \phi_{n}\right\|_{2}^{2}-\left\|\nabla \phi_{n}^{1}\right\|_{2}^{2}-\left\|\nabla \phi_{n}^{2}\right\|_{2}^{2}\right| \leq C \epsilon \\
& \limsup \sup _{n \rightarrow \infty}\left(\left|\gamma-\int_{\mathbb{R}^{2}} \rho\left(\phi_{n}^{1}\right) d \mathbf{x}\right|+\left|\left(\sigma_{0}-\gamma\right)-\int_{\mathbb{R}^{2}} \rho\left(\phi_{n}^{2}\right) d \mathbf{x}\right|\right) \leq \epsilon .
\end{aligned}
$$

The first remark (passing to a subsequence) is that

$$
\lim _{n \rightarrow \infty}\left(\int_{\mathbb{R}^{2}}\left(\rho\left(\phi_{n}\right)-\rho\left(\phi_{n}^{1}\right)-\rho\left(\phi_{n}^{2}\right)\right) d \mathbf{x}\right)=0,
$$

since we have that

$$
\lim _{n \rightarrow \infty}\left|\int_{\mathbb{R}^{2}}\left(\rho\left(\phi_{n}\right)-\rho\left(\phi_{n}^{1}\right)-\rho\left(\phi_{n}^{2}\right)\right) d \mathbf{x}\right| \leq \lim _{n \rightarrow \infty}\left|\gamma-\int_{\mathbb{R}^{2}} \rho\left(\phi_{n}^{1}\right) d \mathbf{x}\right|+\left|\left(\sigma_{0}-\gamma\right)-\int_{\mathbb{R}^{2}} \rho\left(\phi_{n}^{2}\right) d \mathbf{x}\right| .
$$

In particular, we have that $\phi_{n}^{k} \neq 0$ for $k=1,2$. Now, from 15$)$, and using that $j(\mu)=I_{0}$ for any $\mu \in \mathbb{R}$, we have that

$$
\begin{aligned}
I_{0}+\epsilon & >\frac{1}{2} T\left(\phi_{n}\right) \\
& \geq \frac{1}{2}\left(T\left(\phi_{n}^{1}\right)+T\left(\phi_{n}^{2}\right)\right)-\frac{\epsilon C}{2} \\
& \geq j\left(V\left(\phi_{n}^{1}\right)\right)+j\left(V\left(\phi_{n}^{2}\right)\right)-\frac{\epsilon C}{2} \\
& \geq I_{0}+I_{0}-\frac{\epsilon C}{2}
\end{aligned}
$$

implying that $I_{0} \geq 2 I_{0}-\frac{\epsilon C}{2}$, but this is a contradiction if $\epsilon>0$ is small enough. In other words, we have ruled out Dichotomy, meaning that we have Compactness. From this fact, there is a sequence $\left(y_{n}\right)_{n} \subset \mathbb{R}^{2}$ such that for a given $\epsilon>0$, there exists $R_{0} \geq \frac{1}{\epsilon}$ such that

$$
\int_{A(n)} \rho\left(\phi_{n}\right) d \mathbf{x} \leq \epsilon
$$

where $A(n)=\mathbb{R}^{2} \backslash B_{R_{0}}\left(y_{n}\right)$ with $B_{R_{0}}\left(y_{n}\right)$ being the open ball of radius $R_{0}$ around $y_{n}$. If we set $\tilde{\phi}_{n}(x)=\phi_{n}\left(x-y_{n}\right)$, then we have that $\tilde{\phi}_{n} \rightarrow \tilde{\phi}_{0}$ in $H^{1}\left(\mathbb{R}^{2}\right)$. Moreover, we also have that $\partial_{l} \tilde{\phi}_{n} \rightarrow \partial_{l} \tilde{\phi}_{0}$ a. e. in $L^{2}\left(\mathbb{R}^{2}\right)$ for $l=0,1,2$ and $\tilde{\phi}_{n} \rightarrow \tilde{\phi}_{0}$ a. e. in $L^{2}\left(\mathbb{R}^{2}\right)$ for $p \geq 2$. From the compactness condition 17 , we have for $n$ large enough that,

$$
\int_{A(0)}\left|\nabla \tilde{\phi}_{n}\right|^{2} d \mathbf{x} \leq 2 \epsilon, \quad \int_{A(0)}\left|\tilde{\phi}_{n}\right|^{2} d \mathbf{x} \leq 2 \epsilon
$$

On the other hand, from the Sobolev inequality, we conclude for $q \geq 2$ that

$$
\int_{A(0)}\left|\tilde{\phi}_{0}\right|^{q} d \mathbf{x} \leq \epsilon^{q}, \quad A(0)=\mathbb{R}^{2} \backslash B_{R_{0}}(0,0) .
$$

Now, from Fatuo's Lemma for $q \geq 2$ we have that

$$
\begin{aligned}
\int_{\mathbb{R}^{2}}\left|\tilde{\phi}_{0}\right|^{q} d \mathbf{x} & \leq \liminf _{m \rightarrow \infty} \int_{\mathbb{R}^{2}}\left|\tilde{\phi}_{n}\right|^{q} d \mathbf{x} \\
& \leq \liminf _{m \rightarrow \infty} \int_{B_{R_{0}}(0)}\left|\tilde{\phi}_{n}\right|^{q} d \mathbf{x}+2 \epsilon \\
& =\int_{B_{R}(0)}\left|\tilde{\phi}_{0}\right|^{q} d \mathbf{x}+2 \epsilon \\
& \leq \int_{\mathbb{R}^{2}}\left|\tilde{\phi}_{0}\right|^{q} d \mathbf{x}+2 \epsilon
\end{aligned}
$$


where we are using that the embedding $H^{1}\left(B_{R_{0}}(0)\right) \hookrightarrow L^{q}\left(B_{R_{0}}(0)\right)$ for $1 \leq q<\infty$ is compact. From this, we have that $\tilde{\phi}_{n} \rightarrow \tilde{\phi}_{0}$ in $L^{q}\left(\mathbb{R}^{2}\right)$ for $1 \leq q<\infty$, since we have weak convergence $\left(\tilde{\phi}_{n} \rightarrow \tilde{\phi}_{0}\right.$ in $\left.L^{q}\left(\mathbb{R}^{2}\right)\right)$ and the convergence of the norms $\left(\left\|\tilde{\phi}_{n}\right\|_{L^{q}\left(\mathbb{R}^{2}\right)} \rightarrow\left\|\tilde{\phi}_{0}\right\|_{L^{q}\left(\mathbb{R}^{2}\right)}\right)$. From this fact, we conclude that

$$
\lim _{n \rightarrow \infty} V\left(\tilde{\phi}_{n}\right)=V\left(\tilde{\phi}_{0}\right)
$$

meaning that $\tilde{\varphi}_{0} \in \Sigma_{0}$. Moreover, again from Fatou's lemma and the compactness of the embedding $H^{1}\left(B_{R_{0}}(0)\right) \hookrightarrow$ $L^{q}\left(B_{R_{0}}(0)\right)$ for $2 \leq q<\infty$, we also have that

$$
\begin{aligned}
\int_{\mathbb{R}^{2}} \rho\left(\tilde{\varphi}_{0}\right) d \mathbf{x} & \leq \liminf \int_{\mathbb{R}^{2}} \rho\left(\tilde{\varphi}_{n}\right) d \mathbf{x} \\
& \left.\leq\left.\liminf \left(T\left(\tilde{\varphi}_{n}\right)+\int_{\mathbb{R}^{2}} \mid \tilde{\varphi}_{n}\right)\right|^{2} d \mathbf{x}\right) \\
& \leq 2 j(\mu)+\int_{\mathbb{R}^{2}}\left|\tilde{\varphi}_{0}\right|^{2} d \mathbf{x},
\end{aligned}
$$

which implies that

$$
T\left(\tilde{\varphi}_{0}\right)=2 j(\mu)
$$

Now, we will see that $X \neq \emptyset$. In fact, let $\psi \in \Sigma_{0}$ be a solution of the minimization problem (8). Then, there is a Lagrange multiplier $\lambda$ such that for any $v \in H^{1}\left(\mathbb{R}^{2}\right)$

$$
\langle\delta T(\psi), v\rangle=\lambda\langle\delta V(\psi), v\rangle,
$$

where the pairing $\langle\cdot, \cdot\rangle$ is between the spaces $H^{-1}\left(\mathbb{R}^{2}\right)$ and $H^{1}\left(\mathbb{R}^{2}\right)$. In other words, $\psi$ satisfies the equation

$$
-\Delta \psi=\lambda(c \psi+g(\psi))
$$

We see directly that $\lambda \neq 0$. Now, we claim that $\lambda>0$. In fact, let $v \in H^{1}\left(\mathbb{R}^{2}\right)$ such that $\langle\delta V(\psi), v\rangle<0$ and take $t \in \mathbb{R}$. Then we see directly that

$$
\begin{aligned}
& V(\psi+t v)=V(\psi)+\int_{0}^{t}\langle\delta V(\psi+s v), v\rangle d s=\int_{0}^{t}\langle\delta V(\psi+s v), v\rangle d s \\
& T(\psi+t v)=T(\psi)+2 t\langle\delta T(\psi), v\rangle+t^{2} T(v)=T(\psi)+2 t \lambda\langle\delta V(\psi), v\rangle+t^{2} T(v) .
\end{aligned}
$$

If we assume that $\lambda<0$ and take $t$ small enough but negative, then form the continuity of $T$ and $V$, we conclude that $T(\psi+t v)<T(\psi)$ with $V(\psi+t v)<0$, which contradicts the second characterization of the minimization problem (Theorem 2.2). So, we see that $\psi_{\lambda}(x)=\psi(y)$ with $x=\sqrt{\lambda} y$ satisfies the travelling wave equation 2 and $\psi_{\lambda} \in X$.

In the case $N=3$, we use the embedding $H^{1}(U) \subset L^{q}(U)$ is compact, for $2<q<6$ and $U \subset \mathbb{R}^{3}$ bounded, the concentration-compactness, the variational characterization $j$, the conditions (G1)-(G3), and the additional condition on $G$,

$\left[(\right.$ G5) $]$ There is a continuous map $G_{0}: H^{1}\left(\mathbb{R}^{3}\right) \times H^{1}\left(\mathbb{R}^{3}\right) \rightarrow \mathbb{R}$ such that

a) If either $\psi=0$ or $\phi=0$, then $G_{0}(\phi, \psi)(x)=0$,

b)

$$
\begin{aligned}
\int_{\mathbb{R}^{3}} G(\phi+\psi)(z) d \mathbf{x} & =\int_{\mathbb{R}^{3}} G(\phi)(z) d \mathbf{x}+\int_{\mathbb{R}^{3}} G(\psi)(z) d \mathbf{x}+\int_{\mathbb{R}^{3}} G_{0}(\phi, \psi) d \mathbf{x} \\
\int_{\mathbb{R}^{3}} G_{0}(\phi, \psi)(z) d \mathbf{x} & =\int_{\text {supp } \phi} G_{0}(\phi, \psi) d \mathbf{x}=\int_{\mathbb{R}^{3}} G_{0}(\psi, \phi) d \mathbf{x}
\end{aligned}
$$

whenever supp $(\phi) \cap \operatorname{supp}(\psi)=\emptyset$, and

c) For any bounded sequence $\left(\psi_{n}, \phi_{n}\right)_{n} \subset H^{1}\left(\mathbb{R}^{3}\right) \times H^{1}\left(\mathbb{R}^{3}\right)$, the sequence $\left(G_{0}\left(\psi_{n}, \phi_{n}\right)\right)_{n}$ converges. 
Theorem 3.2 (i) $X=\left\{\psi \in H^{1}\left(\mathbb{R}^{3}\right): \psi \neq 0\right.$, $\psi$ solves $\left.(2)\right\}$ has a function.

(ii) There is $\mu_{0}<0$ such that $\varphi \in X$ if and only if $\varphi$ solves the minimization problem

$$
\left\{\begin{aligned}
\varphi & \in \Sigma_{\mu_{0}} \\
T(\varphi) & =\inf \left\{T(\psi): V(\psi)=\mu_{0}\right\}
\end{aligned}\right.
$$

Proof. Let $j(\mu)$ be defined in (5). We want to show that the problem 21) has a solution. In fact, let $\left(\phi_{n}\right)_{n} \subset \Sigma_{\mu_{0}}$ such that

$$
I_{\mu_{0}}=\inf \left\{T\left(\phi_{n}\right): V\left(\phi_{n}\right)=\mu_{0}\right\}=\lim _{n \rightarrow \infty} T\left(\phi_{n}\right) .
$$

We claim that the sequence $\left(\left\|\phi_{n}\right\|_{2}\right)_{n}$ is bounded. If not, assume that for some subsequence, we have that $\left\|\phi_{n_{l}}\right\|_{2} \rightarrow \infty$, as $l \rightarrow \infty$. So, from the Gagliardo-Nirenberg-Sobolev inequality and that the sequence $\left(\left\|\nabla \phi_{n}\right\|_{2}\right)_{n}$ is bounded, we conclude that

$$
0<\frac{c}{2}+\lim _{l \rightarrow \infty}\left(\frac{-\mu_{0}}{\left\|\phi_{n_{l}}\right\|_{2}}\right)=\left|\lim _{l \rightarrow \infty}\left(\frac{\int_{\mathbb{R}^{3}} G\left(\phi_{n_{l}}\right) d \mathbf{x}}{\left\|\phi_{n_{l}}\right\|_{2}}\right)\right| \leq M \lim _{l \rightarrow \infty} \sum_{j=1}^{k}\left(\frac{\left\|\nabla \phi_{n_{l}}\right\|_{2}^{\frac{3 r_{j}}{2}}}{\left\|\phi_{n_{l}}\right\|_{2}^{\frac{r_{j}}{2}}}\right)=0
$$

which is a contradiction, meaning that the sequence $\left(\varphi_{n}\right)_{n}$ is bounded in $H^{1}\left(\mathbb{R}^{3}\right)$. From this, there is $\phi_{0} \in H^{1}\left(\mathbb{R}^{3}\right)$ such that, for a subsequence (denoted the same), if necessary, we have that $\phi_{n} \rightarrow \phi_{0}$ (weakly) in $H^{1}\left(\mathbb{R}^{3}\right)$

Now, we set the measure $\nu_{n}$ with density $\rho\left(\phi_{n}\right)$ with respect to the Lebesgue measure given by

$$
\rho(\phi)=|\nabla \phi|^{2}+|\phi|^{2}
$$

which implies that

$$
\int_{\mathbb{R}^{3}} d \nu_{n}=\int_{\mathbb{R}^{3}} \rho\left(\phi_{n}\right) d \mathbf{x}:=\sigma_{n} \rightarrow \sigma_{0}, \quad n \rightarrow \infty
$$

So, we have that $\sigma_{0} \geq \frac{2}{\beta} j\left(\mu_{0}\right)=-\mu_{0}^{\frac{1}{3}} I$. We now apply the Lions' Concentration-Compactness Principle (see [9]-[10]). First,we see that vanishing is not possible. In fact, as in previous result, covering $\mathbb{R}^{3}$ with a sequence of open boxes $J_{k}$ in such a way that $J_{k} \cap J_{m}=\emptyset$, then we see that

$$
\left\|\phi_{n}\right\|_{r_{j}+2}^{r_{j}+2} \leq\left. C\left(\sup _{k} \int_{J_{k}}\left(\left|\nabla \phi_{n}\right|^{2}+\left|\phi_{n}\right|^{2}\right) d \mathbf{x}\right)^{\frac{r_{j}}{2}}|| \phi_{n}\right|_{H^{1}\left(\mathbb{R}^{3}\right)} ^{2} .
$$

which implies, in the case we have vanishing, that $\left\|\phi_{n}\right\|_{r_{j}+2} \rightarrow 0$, as $n \rightarrow \infty$ for $0<r_{j}<2$. On the other hand, from $V\left(\phi_{n}\right)=\mu_{0}$ and condition (G2), we conclude that

$$
0<\frac{c}{2}\left\|\left.\phi_{n}\right|_{2} ^{2}-\mu_{0} \leq M \sum_{j=1}^{k}\right\| \phi_{n} \|\left._{r^{\frac{3 r_{j}}{2}}}|| \phi_{n}\right|_{2} ^{2-\frac{r_{j}}{2}}
$$

which gives us a contradiction.

If we assume Dichotomy, then there is $0<\gamma<\sigma_{0}$ such that for a given $\epsilon>0$, there exist $R_{0}>0$, a sequence $\left(y_{n}\right)_{n} \subset \mathbb{R}^{3}$, $R_{n} \uparrow+\infty$, and a bounded sequence $\left(\phi_{n}^{i}\right)_{n} \subset H^{1}\left(\mathbb{R}^{3}\right)$ for $i=1,2$ (all depending on $\epsilon$ ) such that

$$
\begin{gathered}
\operatorname{supp}\left(\phi_{n}^{1}\right) \subset B_{R_{0}}\left(x_{n}\right), \quad \operatorname{supp}\left(\phi_{n}^{2}\right) \subset \mathbb{R}^{3} \backslash B_{R_{n}}\left(x_{n}\right) \\
\left\|\phi_{n}-\phi_{n}^{1}-\phi_{n}^{2}\right\|_{H^{1}\left(\mathbb{R}^{3}\right) \leq \epsilon} \\
\left|\left\|\nabla \phi_{n}\right\|_{2}^{2}-\left\|\nabla \phi_{n}^{1}\right\|_{2}^{2}-\left\|\nabla \phi_{n}^{2}\right\|_{2}^{2}\right| \leq C \epsilon \\
\lim \sup _{n \rightarrow \infty}\left(\left|\gamma-\int_{\mathbb{R}^{3}} \rho\left(\phi_{n}^{1}\right) d \mathbf{x}\right|+\left|\left(\sigma_{0}-\gamma\right)-\int_{\mathbb{R}^{3}} \rho\left(\phi_{n}^{2}\right) d \mathbf{x}\right|\right) \leq \epsilon .
\end{gathered}
$$


The first remark (passing to a subsequence) is that

$$
\lim _{n \rightarrow \infty}\left(\int_{\mathbb{R}^{3}}\left(\rho\left(\phi_{n}\right)-\rho\left(\phi_{n}^{1}\right)-\rho\left(\phi_{n}^{2}\right)\right) d \mathbf{x}\right)=0
$$

since we have that

$$
\lim _{n \rightarrow \infty}\left|\int_{\mathbb{R}^{3}}\left(\rho\left(\phi_{n}\right)-\rho\left(\phi_{n}^{1}\right)-\rho\left(\phi_{n}^{2}\right)\right) d \mathbf{x}\right| \leq \lim _{n \rightarrow \infty}\left[\left|\gamma-\int_{\mathbb{R}^{3}} \rho\left(\phi_{n}^{1}\right) d \mathbf{x}\right|+\left|\left(\sigma_{0}-\gamma\right)-\int_{\mathbb{R}^{3}} \rho\left(\phi_{n}^{2}\right) d \mathbf{x}\right|\right] .
$$

Passing to a subsequence, if necessary, we may assume for $i=1,2$ that

$$
\lim _{n \rightarrow \infty}\left\|\phi_{n}^{i}\right\|_{H^{1}\left(\mathbb{R}^{3}\right)}^{2}=\sigma_{i}(\epsilon)>0, \quad \lim _{n \rightarrow \infty} V\left(\phi_{n}^{i}\right)=\mu_{i}(\epsilon)
$$

In particular, form 22, we have that

$$
\sigma_{1}(\epsilon)+\sigma_{2}(\epsilon) \geq \sigma_{0}>0
$$

From the fact that $V \in C^{1}\left(H^{1}\left(\mathbb{R}^{3}\right)\right)$, we easily see that

$$
\left|V\left(\phi_{n}\right)-V\left(\phi_{n}^{1}+\phi_{n}^{2}\right)\right| \leq C\left(\left\|\phi_{n}\right\|_{H^{1}}\right)\left\|\phi_{n}-\left(\phi_{n}^{1}+\phi_{n}^{2}\right)\right\|_{H^{1}\left(\mathbb{R}^{3}\right)},
$$

using that

$$
V(\phi)-V(\psi)=\int_{0}^{1}\langle\delta V(s \phi+(1-s) \psi), \phi-\psi\rangle_{H^{-1}, H^{1}} d s .
$$

From the hypothesis (G5), we have that

$$
V\left(\phi_{n}\right)-V\left(\phi_{n}^{1}\right)-V\left(\phi_{n}^{2}\right)=V\left(\phi_{n}\right)-V\left(\phi_{n}^{1}+\phi_{n}^{2}\right)+\int_{\mathbb{R}^{3}} G_{0}\left(\phi_{n}^{1}, \phi_{n}^{2}\right)(z) d \mathbf{x} .
$$

Now, if we set $\tilde{\phi}_{n}^{i}(x)=\phi_{n}^{i}\left(x-y_{n}\right)$, then we note

$$
\int_{\mathbb{R}^{3}} G_{0}\left(\phi_{n}^{1}, \phi_{n}^{2}\right) d \mathbf{x}=\int_{B_{n}^{c}} G_{0}\left(\tilde{\phi}_{n}^{1}, \tilde{\phi}_{n}^{2}\right)(\mathbf{x}) d \mathbf{x}=\int_{\mathbb{R}^{3}} \chi_{B_{n}^{c}} G_{0}\left(\tilde{\phi}_{n}^{1}, \tilde{\phi}_{n}^{2}\right)(\mathbf{x}) d \mathbf{x}
$$

where $\chi_{A}$ denotes the characteristic function on the set $A$ and $B_{n}=B_{R_{n}}(0)$. On the other hand, the embedding $H^{1}\left(B_{R_{0}}\right) \subset L^{q}\left(B_{R_{0}}\right)$ is compact, for $2<q<6$. So, using that $\left(\tilde{\phi}_{n}^{1}\right)_{n}$ is bounded in $H^{1}\left(\mathbb{R}^{3}\right)$, there is $\phi_{0}^{1} \in H^{1}\left(\mathbb{R}^{3}\right)$ such that $\tilde{\phi}_{n}^{1} \rightarrow \phi_{0}^{1}$ in $L^{q}\left(B_{R_{0}}\right)$ for $2<q<6$. Moreover, for any $\psi \in H^{1}\left(\mathbb{R}^{3}\right)$ we have that $G_{0}\left(\psi, \tilde{\phi}_{n}^{1}\right) \rightarrow G_{0}\left(\psi, \phi_{0}^{1}\right)$ and also that $\chi_{B_{n}^{c}} G_{0}\left(\tilde{\phi}_{n}^{1}, \tilde{\phi}_{n}^{2}\right) \rightarrow 0$ almost everywhere, then the Lebesgue convergence theorem implies that

$$
\lim _{n \rightarrow \infty} \int_{\mathbb{R}^{3}} G_{1}\left(\phi_{n}^{1}, \phi_{n}^{2}\right) d \mathbf{x}=\lim _{n \rightarrow \infty} \int_{\mathbb{R}^{3}} \chi_{B_{n}^{c}} G_{1}\left(\tilde{\phi}_{n}^{1}, \tilde{\phi}_{n}^{2}\right)(\mathbf{x}) d \mathbf{x}=0 .
$$

From the estimates 23 and 24 , we conclude for $n$ large enough that

$$
\left|V\left(\phi_{n}\right)-V\left(\phi_{n}^{1}\right)-V\left(\phi_{n}^{2}\right)\right| \leq \delta(\epsilon)
$$

where $\lim _{\epsilon \rightarrow 0^{+}} \delta(\epsilon)=0$. So, taking limit as $n \rightarrow \infty$, we see that

$$
\left|\mu_{0}-\left(\mu_{1}(\epsilon)+\mu_{2}(\epsilon)\right)\right| \leq \delta(\epsilon)
$$

Assume that $\lim _{\epsilon \rightarrow 0^{+}} \mu_{1}(\epsilon) \geq 0$, then we conclude that

$$
\mu_{2}=\lim _{\epsilon \rightarrow 0^{+}} \mu_{2}(\epsilon)<0
$$

Now, we note that

$$
\left\|\phi_{n}^{1}\right\|_{2}^{2}=\int_{B_{R_{0}}\left(y_{n}\right)}\left|\phi_{n}^{1}\right|^{2} d \mathbf{x} \leq C\left(R_{0}, r\right)\left\|\phi_{n}^{1}\right\|_{r+2}^{2}
$$


Now, from the Gagliardo-Nirenberg-Sobolev inequality for $0<r_{j}<4$, we have that

$$
\left\|\phi_{n}^{1}\right\|_{r_{j}+2}^{r_{j}+2} \leq C\left(r_{j}\right)\left\|\nabla \phi_{n}^{1}\right\|_{2}^{\frac{3 r_{j}}{2}}\left\|\phi_{n}^{1}\right\|_{2}^{2-\frac{r_{j}}{2}} \leq C\left(r_{j}, R_{0}\right)\left\|\nabla \phi_{n}^{1}\right\|_{2}^{\frac{3 r_{j}}{2}}\left\|\phi_{n}^{1}\right\|_{r_{j}+2}^{2-\frac{r_{j}}{2}}
$$

If for a subsequence of $\left(\phi_{n}\right)_{n}$ (denoted the same) we have that $\lim _{n \rightarrow \infty} T\left(\phi_{n}\right)=0$, then we conclude that $\left\|\phi_{n}^{1}\right\|_{r_{j}+2} \rightarrow 0$, as $n \rightarrow \infty$, which implies that $\lim _{n \rightarrow \infty} V\left(\phi_{n}^{1}\right)=0$. In other words, there is $M_{1}>0$ such that $T\left(\phi_{n}\right) \geq T\left(\phi_{n}^{1}\right)>M_{1}$ with $M_{1}$ independent of $\epsilon$ and $n$. Moreover,

$$
j\left(\mu_{0}\right)=\lim _{n \rightarrow \infty} \frac{1}{2} T\left(\phi_{n}\right)>\frac{1}{2} M_{1}+\liminf _{n \rightarrow \infty} \frac{1}{2} T\left(\phi_{n}^{2}\right)-\epsilon C>\frac{1}{2} M_{1}+j\left(\mu_{2}(\epsilon)\right)-\epsilon C,
$$

which implies from Lemma 2.1), after taking $\epsilon \rightarrow 0$, that

$$
\left(-\mu_{0}\right)^{\frac{1}{3}} I \geq \frac{1}{2} M_{1}+\left(-\mu_{2}\right)^{\frac{1}{3}} I \geq \frac{1}{2} M_{1}+\left(-\mu_{0}\right)^{\frac{1}{3}} I,
$$

but this is a contradiction since $0>\mu_{0} \geq \mu_{2}$. So, we may assume that $\mu_{i}(\epsilon) \leq 0$ for $i=1,2$. Then, from 15 , we have that

$$
\begin{aligned}
j\left(\mu_{0}\right)+\epsilon & \geq \frac{1}{2}\left(T\left(\phi_{n, 1}\right)+T\left(\phi_{n, 1}\right)\right)-\frac{\epsilon C}{2} \\
& \geq j\left(V\left(\phi_{n, 1}\right)\right)+j\left(V\left(\phi_{n, 2}\right)\right)-\frac{\epsilon C}{2} \\
& \geq j\left(\mu_{1}(\epsilon)\right)+j\left(\mu_{2}(\epsilon)\right)-\frac{\epsilon C}{2},
\end{aligned}
$$

which implies from Lemma 2.1), after taking $\epsilon \rightarrow 0$, that

$$
\left(\left(-\mu_{1}\right)+\left(-\mu_{2}\right)\right)^{\frac{1}{3}}=\left(-\mu_{0}\right)^{\frac{1}{3}} \geq\left(-\mu_{1}\right)^{\frac{1}{3}}+\left(-\mu_{2}\right)^{\frac{1}{3}},
$$

but this contradicts the fact that the function $f(t)=t^{\frac{1}{3}}$ is strictly concave for $t \in \mathbb{R}^{+}$, since we have that

$$
f\left(t_{1}+t_{2}\right)<f\left(t_{1}\right)+f\left(t_{2}\right), \text { for } t_{1}, t_{2}>0
$$

So, we have ruled out Dichotomy. Using the compactness property as in the case $N=2$, we conclude that there is a minimizer $\varphi_{0} \in H^{1}\left(\mathbb{R}^{3}\right)$ for $j\left(\mu_{0}\right)$,

$$
T\left(\varphi_{0}\right)=2 j(\mu)
$$

Now, we will see that $X \neq \emptyset$. In fact, let $\psi \in \Sigma_{\mu_{0}}$ be a solution of the minimization problem (10). Then, there is a Lagrange multiplier $\lambda$ such that for any $v \in H^{1}\left(\mathbb{R}^{2}\right)$

$$
\langle\delta T(\psi), v\rangle=\lambda\langle\delta V(\psi), v\rangle
$$

where the pairing $\langle\cdot, \cdot\rangle$ is between the spaces $H^{-1}\left(\mathbb{R}^{3}\right)-H^{1}\left(\mathbb{R}^{3}\right)$. In other words, $\psi$ satisfies the equation

$$
-\Delta \psi=\lambda(c \psi+g(\psi))
$$

We see directly that $\lambda \neq 0$. Now, we claim that $\lambda>0$. In fact, let $v \in H^{1}\left(\mathbb{R}^{3}\right)$ such that $\langle\delta V(\psi), v\rangle<0$ and take $t \in \mathbb{R}$. Then we see directly that

$$
\begin{aligned}
& V(\psi+t v)=V(\psi)+\int_{0}^{t}\langle\delta V(\psi+s v), v\rangle d s=\int_{0}^{t}\langle\delta V(\psi+s v), v\rangle d s \\
& T(\psi+t v)=T(\psi)+2 t\langle\delta T(\psi), v\rangle+t^{2} T(v)=T(\psi)+2 t \lambda\langle\delta V(\psi), v\rangle+t^{2} T(v) .
\end{aligned}
$$

If we assume that $\lambda<0$ and take $t$ small enough but negative, then form the continuity of $T$ and $V$, we conclude that $T(\psi+t v)<T(\psi)$ with $V(\psi+t v)<0$, which contradicts the second characterization of the minimization problem (Theorem (2.2). So, we see that $\psi_{\lambda_{0}}(x)=\psi(y)$ with $x=\sqrt[3]{\lambda_{0}} y$ satisfies the travelling wave equation $(2)$ and $\psi_{\lambda_{0}} \in X$ with $\lambda_{0}=\lambda^{\frac{3}{2}}$. 


\section{Applications}

In this section we provide some examples of operators $g$ satisfying the conditions imposed on $G$. Before we go further, we introduce some notation to be considered. Hereafter, we set

$$
p^{*}= \begin{cases}\infty, & \text { for } N=2 \\ 4, & \text { for } N=3\end{cases}
$$

\subsection{Generalizations of the $\mathrm{KdV}$ and the Gardner equation in $\mathbb{R}^{N}$}

We consider the dispersive model of the form $\mathrm{u}_{t}+\Delta u_{x}-\left(a|u|^{p-1} u-b|u|^{q-1} u\right)_{x}=0$. For this model, the $x$-travelling equation is given by $-c u+\Delta u-\left(a|u|^{p-1} u-b|u|^{q-1} u\right)=0$. In this particular case, we have that

$$
g_{1}(t)=a|t|^{p-1} t-b|t|^{q-1} t
$$

and that $G_{1}$ is given by

$$
G_{1}(t)=\frac{a}{p+2}|t|^{p+2}-\frac{b}{q+2}|t|^{q+2} .
$$

We will establish the existence of travelling waves by imposing some restrictions on the parameters $a$ and $p$. We start defining

$$
\begin{aligned}
& a_{0}(p)=\left\{\begin{array}{ll}
\infty, & \text { for } p<q \\
b, & \text { for } p=q \\
\left(\frac{2(p-q)}{c q(p+2)}\right)^{\frac{p}{p-q}}\left(\frac{(p+2) q b}{(q+2) p}\right)^{\frac{p}{q}}, & \text { for } p>q .
\end{array},\right. \\
& a_{1}(p)=\frac{q b c(p+2)}{c p(q+2)-2(p-q) b}, \quad c>b, \quad p>q .
\end{aligned}
$$

We also define the sets

$$
\mathcal{A}_{c, q, b}=\left\{(p, a): 0<p<p^{*}, \quad a<a_{0}(p)\right\}
$$

and

$$
\mathcal{B}_{c, q, b}=\left\{(p, a): 0<p<p^{*}, \quad a_{1}(p)<a<a_{0}(p)\right\}
$$

for $c>b$ and $p>q$ in the case $a_{1}(p)<a_{0}(p)$.

We are going to verify the conditions (G1)-(G5) for the function $G_{1}$. First note that

$$
\int_{\mathbb{R}^{N}}\left|G_{1}(\phi)\right| d \mathbf{x} \leq\left(\frac{|a|}{p+2}+\frac{|b|}{q+2}\right)\left(\|\phi\|_{p+2}^{p+2}+\|\phi\|_{q+2}^{q+2}\right),
$$

meaning that the condition (G1) holds. Now, note that the condition (G3) holds. In fact,

$$
\begin{aligned}
G_{1}(\lambda t) & =\frac{a \lambda^{p+2}}{p+2}|t|^{p+2}-\frac{b \lambda^{q+2}}{q+2}|t|^{q+2} \\
& =\lambda^{p+2}\left(\frac{a}{p+2}|t|^{p+2}-\frac{b \lambda^{q-p}}{q+2}|t|^{q+2}\right) \\
& =\lambda^{q+2}\left(\frac{a \lambda^{p-q}}{p+2}|t|^{p+2}-\frac{b}{q+2}|t|^{q+2}\right) .
\end{aligned}
$$

Now, we verify the condition (G2) holds for $(p, a) \in \mathcal{A}_{c, q, b}$.

1) Assume that either $p<q$ or $a<0$. Then we have for any non trivial $\phi \in H^{1}\left(\mathbb{R}^{2}\right)$, we define

$$
h_{\phi}(\lambda)=V(\lambda \phi)=\lambda^{2}\left(\frac{c}{2}\|\phi\|_{2}^{2}+\frac{a \lambda^{p}}{p+2}\|\phi\|_{p+2}^{p+2}-\frac{b \lambda^{q}}{q+2}\|\phi\|_{q+2}^{q+2}\right)=\lambda^{2} \tilde{h}_{\phi}(\lambda) .
$$

Then we see that $\tilde{h}_{\phi}(0)>0$ and $\lim _{\lambda \rightarrow \infty} \tilde{h}_{\phi}(\lambda)=-\infty$, where $h_{\phi}(\lambda)=\lambda^{2} \tilde{h}_{\phi}(\lambda)$. So, for $\lambda_{0}>0$ large enough, we have that $\tilde{h}_{\phi}\left(\lambda_{0}\right)<0$. In other words, $\varphi_{0}=\lambda_{0} \phi$ is such that $V\left(\varphi_{0}\right)<0$. 
2) Assume that $p>q$ and take $a>0$. Now, for $\lambda>0$ and a non trivial $\phi \in H^{1}\left(\mathbb{R}^{N}\right)$, we define the function $\phi^{\lambda}$ as $\phi^{\lambda}(x, y)=\lambda^{\frac{1}{4}} \phi(w, z)$, where $w=\lambda x$ and $y=z \in \mathbb{R}^{N-1}$. Then, we have that

$$
V\left(\phi^{\lambda}\right)=\frac{c \lambda^{-\frac{1}{2}}}{2}\|\phi\|_{2}^{2}+\frac{a \lambda^{\frac{p-2}{4}}}{p+2}\|\phi\|_{p+2}^{p+2}-\frac{b \lambda^{\frac{q-2}{4}}}{q+2}\|\phi\|_{q+2}^{q+2}
$$

If we set for $\lambda>0$ the function $g$ defined in $(0, \infty)$ by

$$
g_{\lambda, p, q}(s)=\frac{c \lambda^{-\frac{1}{2}}}{2} s^{2}+\frac{a \lambda^{\frac{p-2}{4}}}{p+2} s^{p+2}-\frac{b \lambda^{\frac{q-2}{4}}}{q+2} s^{q+2}=\lambda^{-\frac{1}{4}} s^{2}\left(\frac{c}{2}+\frac{a\left(\lambda^{\frac{1}{4}} s\right)^{p}}{p+2}-\frac{b\left(\lambda^{\frac{1}{4}} s\right)^{q}}{q+2}\right) .
$$

From this definition, we set the function

$$
\tilde{g}_{\lambda, p, q}(w)=\frac{c}{2}+\frac{a w^{p}}{p+2}-\frac{b w^{q}}{q+2} .
$$

We see directly that there is a unique positive real number $w_{0}$ such that $\frac{d}{d s} \tilde{g}_{\lambda, p, q}\left(w_{0}\right)=0$, with $w_{0}$ given by

$$
w_{0}=\left(\frac{(p+2) q b}{(q+2) p a}\right)^{\frac{1}{p-q}}
$$

Moreover, we also have that

$$
\tilde{g}_{\lambda, p, q}\left(w_{0}\right)=\frac{c}{2}+\frac{a}{p+2}\left(1-\frac{p}{q}\right) w_{0}^{p}=\frac{c}{2}+a^{\frac{-q}{p-q}} \frac{1}{p+2}\left(1-\frac{p}{q}\right)\left(\frac{(p+2) q b}{(q+2) p}\right)^{\frac{p}{p-q}} .
$$

We see that $\tilde{g}_{\lambda, p, q}\left(w_{0}\right)<0$, for $0<a<a_{0}(p, q, c, b)$ by taking

$$
a_{0}=\left(\frac{2(p-q)}{c q}\right)^{\frac{p-q}{q}}\left(\frac{(p+2) q b}{(q+2) p}\right)^{\frac{p}{q}}
$$

which implies that there is $s_{0}>0$ such that $g_{\lambda, p, q}\left(s_{0}\right)<0$, in the case $0<a<a_{0}(p, q, c, b)$.

Now, if we consider $\phi_{R, s}=s \chi_{B_{R}}$, where $\chi_{B_{R}}$ denotes the characteristic function on the ball $B_{R}$ of radius $R$ denoted, then we have that $\left\|\phi_{R, s}\right\|_{r}^{r}=\left|B_{R}\right| s^{r}$ for $r \geq 1$ and that

$$
V\left(\left(\phi_{R, s}\right)^{\lambda}\right)=\left|B_{R}\right| g_{\lambda, p, q}(s)
$$

which implies that $V\left(\left(\phi_{R, s_{0}}\right)^{\lambda}\right)=\left|B_{R}\right| g_{\lambda}\left(s_{0}\right)<0$. By a density argument, we have that there is $\varphi_{0} \in H^{1}\left(\mathbb{R}^{N}\right)$ such that $V\left(\varphi_{0}\right)<0$.

3) Assume that $p=q$ and take $a<b$, Then we see that

$$
\tilde{g}_{\lambda, p, p}(w)=\frac{c}{2}+\frac{(a-b)}{p+2} w^{p},
$$

which implies the existence of $w_{0}>0$ such that $\tilde{g}_{\lambda, p, p}\left(w_{0}\right)<0$, as desired. In other words, we reach the condition (G2).

Now, we verify the condition (G4) for either $(p, a) \in \mathcal{A}_{c, b, q}$ for $p \leq q$ or $(p, a) \in \mathcal{A}_{c, b, q}$ for $c>b$ and $p>q$. So, take $\psi$ such that $V(\psi)=0$ and define the function $\tilde{h}_{\psi}$ by

$$
\tilde{h}_{\psi}(\lambda)=\frac{c}{2}\|\psi\|_{2}^{2}+\frac{a \lambda^{p}}{p+2}\|\psi\|_{p+2}^{p+2}-\frac{b \lambda^{q}}{q+2}\|\psi\|_{q+2}^{q+2} .
$$

Clearly, $\tilde{h}_{\psi}(0)>0$ and $\tilde{h}_{\psi}(1)=V(\psi)=0$. First assume that $a \leq 0$. In this case, we see that $\tilde{h}_{\psi}^{\prime}(\lambda)<0$ for $\lambda>0$ and that $h_{\psi}(\lambda)<0$ for $\lambda>1$. 
On the other hand, in the case $a>0$, we see that $\tilde{h}_{\psi}(1)=V(\psi)=0$ implies that

$$
\frac{a}{(p+2)}\|\psi\|_{p+2}^{p+2}<\frac{b}{q+2}\|\psi\|_{q+2}^{q+2} .
$$

We also conclude that there is a unique $\lambda_{0}>0$ such that

$$
\tilde{h}_{\psi}^{\prime}\left(\lambda_{0}\right)=0 \quad \Leftrightarrow \quad \lambda_{0}^{p-q}=\frac{q}{p} \frac{b(p+2)}{a(q+2)} \frac{\|\psi\|_{q+2}^{q+2}}{\|\psi\|_{p+2}^{p+2}}>\frac{q}{p} .
$$

Moreover, we also have that

$$
\tilde{h}_{\psi}\left(\lambda_{0}\right)=\frac{c}{2}\|\psi\|_{2}^{2}+\frac{b \lambda_{0}^{q}}{p(q+2)}(q-p)\|\psi\|_{q+2}^{q+2} .
$$

So, for $q>p$ we have that $\lambda_{0}<1$, that $\tilde{h}_{\psi}\left(\lambda_{0}\right)>0$, and also that $h_{\psi}(\lambda)<0$ for $\lambda>1$.

Assume now that $p>q$ and $a>0$. In this case, we have that there is a unique $\lambda_{0}>0$ given by (26). If we had $\lambda_{0}=1$, then we conclude that

$$
\frac{\|\psi\|_{p+2}^{p+2}}{\|\psi\|_{q+2}^{q+2}}=\frac{q b(p+2)}{p a(q+2)}, \quad \frac{\|\psi\|_{2}^{2}}{\|\psi\|_{q+2}^{q+2}}=\frac{2 b(p-q)}{p c(q+2)} .
$$

On the other hand, we also have for $2<q<p$ that

$$
\|\psi\|_{q+2}^{q+2}=\left(\int_{|\psi| \leq 1}+\int_{|\psi| \geq 1}\right)|\psi(z)|^{q+2} d \mathbf{x} \leq\|\psi\|_{2}^{2}+\|\psi\|_{p+2}^{p+2} .
$$

which implies that

$$
1 \leq \frac{q b(p+2)}{p a(q+2)}+\frac{2 b(p-q)}{p c(q+2)} \quad \Leftrightarrow \quad a(c p(q+2)-2(p-q) b)<q b c(p+2) .
$$

If we assume for example that $c>b$, and choose $a>0$ such that

$$
a \geq \frac{q b c(p+2)}{c p(q+2)-2(p-q) b},
$$

then we conclude that $\lambda_{0} \neq 1$. Now, if we had $\lambda_{0}<1$ and $a>0$ satisfies (27), then from (26) we see that

$$
p \lambda_{0}^{p} \geq q \lambda_{0}^{q}
$$

meaning that the function $w(p)=p \lambda_{0}^{p}$ is an increasing function, but this happens, only if $1+p \ln \left(\lambda_{0}\right)>0$, which requires $\lambda_{0}>1$, but we are assuming that $\lambda_{0}<1$. In other words, under the assumption that $a>0$ satisfies $(27)$, we have necessarily that $\lambda_{0}>1$, and so, we see directly that $h_{\psi}(\lambda)<0$ for $\lambda>1$, but close to $1^{+}$. Finally, the case $p=q$ and $a<b$ follows trivially.

Finally, we note that condition (G5) is trivially obtained.

Now, we consider the model

$\mathrm{u}_{t}+\Delta u_{x}-\left(a|u|^{p-1} u-b u^{q}\right)_{x}=0$, which the $x$-travelling equation is given by $-c u+\Delta u-\left(a|u|^{p-1} u-b u^{q}\right)=0$. So, we have that

$$
\left.g_{2} t\right)=a|t|^{p-1} t-b t^{q},
$$

and that $G_{2}$ is given by

$$
G_{2}(t)=\frac{a}{p+2}|t|^{p+2}-\frac{b}{q+2} t^{q+2} .
$$


In order to obtain similar results as in previous case, we need to adjust some issues. For instance, we take $q>2, b>0$ and $(p, a) \in \mathcal{C}_{c, q, b}$, where

$$
\mathcal{C}_{c, q, b}=\left\{(p, a): 0<p<p^{*}, \quad a_{21}(p)<a<a_{22}(p)\right\}
$$

and

$$
a_{22}=\left\{\begin{array}{ll}
\infty, & \text { for } p<q \\
\left(\frac{2(p-q)}{c q(p+2)}\right)^{\frac{p}{p-q}}\left(\frac{(p+2) q b}{(q+2) p}\right)^{\frac{p}{q}}, & \text { for } p>q .
\end{array},\right.
$$

First, we have that $G_{2}$ is given by

$$
G_{2}(t)=\frac{a}{p+2}|t|^{p+2}-\frac{b}{q+2} t^{q+2} .
$$

As in the case above, the conditions (G1) and (G3) hold using the same argument.

We now verify the condition (G2) holds for $(p, a) \in \mathcal{A}_{c, b, q}$ with $p \neq q$.

1) Assume that either $p<q$ or $a<0$. Recall that for $\phi \in H^{1}\left(\mathbb{R}^{N}\right)$, we define the function

$$
\tilde{h}_{\phi}(\lambda)=\frac{c}{2}\|\phi\|_{2}^{2}+\frac{a \lambda^{p}}{p+2}\|\phi\|_{p+2}^{p+2}-\frac{b \lambda^{q}}{q+2} \int_{\mathbb{R}^{N}} \phi^{q+2}(\mathbf{x}) d \mathbf{x} .
$$

If we choose $\phi$ in such a way that

$$
\int_{\mathbb{R}^{N}} \phi^{q+2} d \mathbf{x}>0
$$

we have that $\tilde{h}_{\phi}(0)>0$ and $\lim _{\lambda \rightarrow \infty} \tilde{h}_{\phi}(\lambda)=-\infty$. So, for $\lambda_{0}>0$ large enough, we have that $\tilde{h}_{\phi}\left(\lambda_{0}\right)<0$. In other words, $\varphi_{0}=\lambda_{0} \phi$ is such that $V\left(\varphi_{0}\right)<0$.

2) Assume that $p>q$. If $\phi \in H^{1}\left(\mathbb{R}^{N}\right)$ is non trivial, we define $\phi^{\lambda}$ for $\lambda>0$ as the function $\phi^{\lambda}(x, y)=\lambda^{\frac{1}{4}} \phi(z, w)$ where $x=\lambda w$ and $y=z \in \mathbb{R}^{N-1}$. Then, we have that

$$
V\left(\phi^{\lambda}\right)=\frac{c \lambda^{-\frac{1}{2}}}{2}\|\phi\|_{2}^{2}+\frac{a \lambda^{\frac{p-2}{4}}}{p+2}\|\phi\|_{p+2}^{p+2}-\frac{b \lambda^{\frac{q-2}{4}}}{q+2} \int_{\mathbb{R}^{N}} \phi^{q+2} d \mathbf{x}
$$

As done for $G_{1}$, if for $\lambda>0$ we set the function $g_{\lambda, p, q}$ defined in $(0, \infty)$ by

$$
g_{\lambda, p, q}(s)=\frac{c \lambda^{-\frac{1}{2}}}{2} s^{2}+\frac{a \lambda^{\frac{p-4}{2}}}{p+2} s^{p+2}-\frac{b \lambda^{\frac{q-4}{2}}}{q+2} s^{q+2} .
$$

From previous case, we know that there is $s_{0}>0$ such that $g_{\lambda, p, q}\left(s_{0}\right)<0$, for $0<a<a_{0}$, meaning that we have the condition (G2) since by a density argument as in previous case, we have that there is $\varphi_{0} \in H^{1}\left(\mathbb{R}^{N}\right)$ such that $V\left(\varphi_{0}\right)<0$.

Now, we establish the validity of the condition $(\mathbf{G} 4)$. In this case, take $\psi$ such that $V(\psi)=0$. We see directly that

$$
\tilde{h}_{\psi}(\lambda)=c\|\psi\|_{2}^{2}+\frac{a \lambda^{p}}{p+2}\|\psi\|_{p+2}^{p+2}-\frac{b \lambda^{q}}{q+2} \int_{\mathbb{R}^{N}} \psi^{q+2} d \mathbf{x} .
$$

Clearly, $\tilde{h}_{\psi}(0)>0$ and $\tilde{h}_{\psi}(1)=V(\psi)=0$. The first remark is that

$$
\tilde{h}_{\psi}^{\prime}\left(\lambda_{0}\right)=0 \Leftrightarrow \frac{b q \int_{\mathbb{R}^{N}} \psi^{q+2} d \mathbf{x}}{q+2} \lambda_{0}^{q}=\frac{a p\|\psi\|_{p+2}^{p+2}}{p+2} \lambda_{0}^{p} .
$$

In the case

$$
a \int_{\mathbb{R}^{N}} \psi^{q+2} d \mathbf{x}<0
$$

we conclude that $\tilde{h}_{\psi}^{\prime}(\lambda)<0$ for $\lambda>0$ and so $\tilde{h}_{\psi}(\lambda)<0$ for $\lambda>1$. 
Now, in the case $a \int_{\mathbb{R}^{N}} \psi^{q+2} d \mathbf{x}>0$, we have that there is a unique $\lambda_{0}>0$ such that

$$
\tilde{h}_{\psi}^{\prime}\left(\lambda_{0}\right)=0 \Leftrightarrow \lambda_{0}^{p-q}=\frac{q}{p} \frac{b(p+2)}{a(q+2)} \frac{\int_{\mathbb{R}^{N}} \psi^{q+2} d \mathbf{x}}{\|\psi\|_{p+2}^{p+2}} .
$$

Moreover, we also have that

$$
\tilde{h}_{\psi}\left(\lambda_{0}\right)=c\|\psi\|_{2}^{2}+\frac{a \lambda_{0}^{p}}{q(p+2)}(q-p)\|\psi\|_{p+2}^{p+2}>0
$$

if we assume $a(q-p)>0$. So, supposing that $a>0$, then we have that $q>p$ and that $\int_{\mathbb{R}^{N}} \psi^{q+2} d \mathbf{x}>0$. In this case, we conclude that

$$
\lambda_{0}^{p-q}=\frac{q}{p} \frac{b(p+2)}{a(q+2)} \frac{\int_{\mathbb{R}^{N}} \psi^{q+2} d \mathbf{x}}{\|\psi\|_{p+2}^{p+2}}>\frac{q}{p},
$$

since we have from the condition $V(\psi)=0$ that

$$
\frac{b(p+2)}{a(q+2)} \frac{\int_{\mathbb{R}^{N}} \psi^{q+2} d \mathbf{x}}{\|\psi\|_{p+2}^{p+2}}>1 .
$$

From this fact, we conclude that $q>p$, and also that $\lambda_{0}<1$. So, from this analysis, we have that $h_{\psi}(\lambda)<0$ for $\lambda>1$, but close to $1^{+}$.

Now, assume that $a<0$, then we have that $p>q$ and that $\int_{\mathbb{R}^{N}} \psi^{q+2} d \mathbf{x}<0$. In this case, we conclude that

$$
\lambda_{0}^{p-q}=\frac{q}{p} \frac{b(p+2)}{a(q+2)} \frac{\int_{\mathbb{R}^{N}} \psi^{q+2} d \mathbf{x}}{\|\psi\|_{p+2}^{p+2}}<\frac{q}{p},
$$

because the condition $V(\psi)=0$ implies that

$$
\frac{b(p+2)}{a(q+2)} \frac{\int_{\mathbb{R}^{N}} \psi^{q+2} d \mathbf{x}}{\|\psi\|_{p+2}^{p+2}}<1
$$

Moreover, we also have that $\tilde{h}_{\psi}\left(\lambda_{0}\right)=c\|\psi\|_{2}^{2}+\frac{a \lambda_{0}^{p}}{q(p+2)}(q-p)\|\psi\|_{p+2}^{p+2}>0$ and also that $\lambda_{0}<1$. So, from this analysis, we have that $h_{\psi}(\lambda)<0$ for $\lambda>1$, but close to $1^{+}$, for $a<0$ and $p>q$.

Finally as in previous case, condition (G5) for $N=2$ follows trivially.

We note that using the same type of arguments as above, we also verify conditions (G1)-(G5) in the case

$$
g_{3}(t)=a t^{2 p+1}-b t^{p+1}=a|t|^{2 p} t-b t^{p+1},
$$

for appropriates $p$ and $a$.

\subsection{Davey-Stewartson type systems}

We consider the standing wave equation for the Davey-Stewartson system in $\mathbb{R}^{N}$

$$
\left\{\begin{aligned}
i u_{t}+\Delta u+b E\left(|u|^{2}\right) u-a|u|^{p} u & =0 \\
-\Delta v & =b_{2}\left(|u|^{2}\right)_{x}
\end{aligned}\right.
$$

where $p>0, b=b_{1} b_{2}>0, a \in \mathbb{R}$, and $E$ is a (non local) linear operator defined via the Fourier transform $\mathcal{F}$ by

$$
\widehat{E}(u)(\xi)=\Gamma_{1}(\xi) \widehat{u}(\xi), \quad \Gamma_{1}(\xi)=\frac{\xi_{1}^{2}}{\|\xi\|^{2}}, \quad \xi \in \mathbb{R}^{N} .
$$


In this case, standing wave equation associated with the Davey-Stewardson system is reduced to find solutions $u$ of the Schrödinge like model, -c $u+\Delta u=a|u|^{p} u-b E\left(|u|^{2}\right) u, \quad u \in H^{1}\left(\mathbb{R}^{N}\right) \backslash\{0\}$. From this model, we have that

$$
g_{4}(u)=a|u|^{p} u-b E\left(|u|^{2}\right) u, \quad G_{4}(u)=\frac{a}{p+2}|u|^{p+2}-\frac{b}{4} E\left(|u|^{2}\right)|u|^{2} .
$$

We will establish the existence of travelling waves by imposing some restrictions on the parameters $a$ and $p$. We start defining

$$
\begin{aligned}
& a_{0}(p)= \begin{cases}\infty, & \text { for } p<2 \\
b, & \text { for } p=2 \\
\left(\frac{p-2}{c(p+2)}\right)^{\frac{p}{p-2}}\left(\frac{(p+2) b}{2 p}\right)^{\frac{p}{2}}, & \text { for } p>2 .\end{cases} \\
& a_{1}(p)=\frac{b c(p+2)}{2 c p-(p-2) b}, \quad c>b, \quad p>2 .
\end{aligned}
$$

We also define the sets

$$
\mathcal{A}_{c, b}=\left\{(p, a): 0<p<p^{*}, \quad a<a_{0}(p)\right\}
$$

and

$$
\mathcal{B}_{c, b}=\left\{(p, a): 0<p<p^{*}, \quad a_{1}(p)<a<a_{0}(p)\right\}
$$

for $c>b$ and $p>2$ in the case $a_{1}(p)<a_{0}(p)$.

The condition (G1)-(G5) are verified in the work by Rolci Cipolatti (see [4]) applied to the operator $-V$ ). For instance, the condition (G1) follows from the Remark (2.2) estimate (2.13) applied to $|\psi|^{2}$.

To verify the condition (G2) for $(p, a) \in \mathcal{A}_{c, b}$, we proceed as in the first example with $q=2$. For instance, assume that $a>0$. So, for a non trivial $\phi \in H^{1}\left(\mathbb{R}^{N}\right)$, we define the function $\phi^{\lambda}$ for $\lambda>0$ as the function $\phi^{\lambda}(x, y)=\lambda^{\frac{1}{4}} \phi(w, z)$ where $w=\lambda x$ and $y=z \in \mathbb{R}^{N-1}$. Then, for $f(\lambda)$ defined as

$$
\begin{aligned}
f(\lambda) & =\int_{\mathbb{R}^{N}} E\left(\left|\phi^{\lambda}\right|^{2}\right)\left|\phi^{\lambda}\right|^{2} d x d y \\
& =\int_{\mathbb{R}^{N}} \sigma_{1}(\xi, \eta)\left|\mathcal{F}\left(\left|\phi^{\lambda}\right|^{2}\right)\right|^{2} d \eta d \xi \\
& =\frac{1}{\lambda} \int_{\mathbb{R}^{N}} \sigma_{1}(\xi, \eta)\left|\mathcal{F}\left(|\phi|^{2}\right)\left(\frac{\xi}{\lambda}, \eta\right)\right|^{2} d \eta d \xi \\
& =\int_{\mathbb{R}^{N}} \sigma_{1}(\lambda \xi, \eta)\left|\mathcal{F}\left(|\phi|^{2}\right)(\xi, \eta)\right|^{2} d \eta d \xi
\end{aligned}
$$

From this expression we see that $f(0)=0$, that $f$ is an increasing function, and that

$$
\lim _{\lambda \rightarrow \infty} f(\lambda)=\|\phi\|_{4}^{4}
$$

From this, given $\epsilon>0$ there is $\lambda>0$ such that

$$
-\frac{b}{4} \int_{\mathbb{R}^{N}} E\left(\left|\phi^{\lambda}\right|^{2}\right)\left|\phi^{\lambda}\right|^{2} d x d y<\epsilon-\frac{b}{4}\|\phi\|_{4}^{4}
$$

Now, if we take $R>0$ and $\phi_{R, s}=s \chi_{B_{R}}$ where $B_{R}$ is the characteristic function of the ball of radius $R$ centered at the origin, we have that $\|\left.\phi_{R, s}\right|_{r} ^{r}=\left|B_{R}\right| s^{r}$ for $r \geq 1$ and for some $\lambda>0$ that

$$
\begin{aligned}
V\left(\left(\phi_{R, s}\right)^{\lambda}\right)-\epsilon & <\frac{c \lambda^{-\frac{1}{2}}}{2}\left\|\phi_{R, s}\right\|_{2}^{2}+\frac{a \lambda \frac{p-2}{4}}{p+2}\left\|\phi_{R, s}\right\|_{q+2}^{q+2}-\frac{b}{4}\left\|\phi_{R, s}\right\|_{4}^{4} \\
& <\lambda^{-\frac{1}{4}} s^{2}\left(\frac{c}{2}+\frac{a\left(\lambda^{\frac{1}{4}} s\right)^{p}}{p+2}-\frac{b\left(\lambda^{\frac{1}{4}} s\right)^{2}}{4}\right)
\end{aligned}
$$

We see that $\tilde{g}_{\lambda, p, 2}\left(w_{0}\right)<0$, for $0<a<a_{0}(p, 2, c, b)$, where

$$
a_{0}=\left(\frac{2(p-2)}{2 c(p+2)}\right)^{\frac{p}{p-2}}\left(\frac{(p+2) 2 b}{4 p}\right)^{\frac{p}{q}}
$$


which implies that there is $s_{0}>0$ such that $g_{\lambda, p, 2}\left(s_{0}\right)<0$, in the case $0<a<a_{0}(p, c, b)$, and also that

$$
V\left(\left(\phi_{R, s_{0}}\right)^{\lambda}\right)-\epsilon<\left|B_{R}\right| g_{\lambda}\left(s_{0}\right)
$$

which implies that $V\left(\left(\phi_{R, s_{0}}\right)^{\lambda}\right)<0$ taking $R>0$ large enough, as desired.

On the other hand, it is straightforward to verify the condition (G4) for either $(p, a) \in \mathcal{A}_{c, b}$ for $p \leq 2$ or $(p, a) \in \mathcal{A}_{c, b}$ for $c>b$ and $p>2$. For non trivial $\psi \in H^{1}\left(\mathbb{R}^{N}\right)$, we define

$$
\tilde{h}_{\psi}(\lambda)=c\|\psi\|_{2}^{2}+\frac{a \lambda^{p}}{p+2}\|\psi\|_{p+2}^{p+2}-\frac{b \lambda^{2}}{4} \int_{\mathbb{R}^{N}} E\left(|\psi|^{2}\right)|\psi|^{2} d \mathbf{x}
$$

Clearly, we for $a \leq 0$, we see that $\tilde{h}_{\psi}^{\prime}(\lambda)<0$ for $\lambda>0$ and that $h_{\psi}(\lambda)<0$ for $\lambda>1$.

On the other hand, in the case $a>0$, we see that $\tilde{h}_{\psi}(1)=V(\psi)=0$ implies that

$$
\frac{a}{(p+2)}\|\psi\|_{p+2}^{p+2}<\frac{b}{4} \int_{\mathbb{R}^{N}} E\left(|\psi|^{2}\right)|\psi|^{2} d \mathbf{x}
$$

We also conclude that there is a unique $\lambda_{0}>0$ such that

$$
\tilde{h}_{\psi}^{\prime}\left(\lambda_{0}\right)=0 \Leftrightarrow \lambda_{0}^{p-2}=\frac{2}{p} \frac{b(p+2)}{4 a} \frac{\int_{\mathbb{R}^{N}} E\left(|\psi|^{2}\right)|\psi|^{2} d \mathbf{x}}{\|\psi\|_{p+2}^{p+2}}>\frac{2}{p} .
$$

Moreover, we also have that

$$
\tilde{h}_{\psi}\left(\lambda_{0}\right)=\frac{c}{2}\|\psi\|_{2}^{2}+\frac{b \lambda_{0}^{2}}{4 p}(2-p) \int_{\mathbb{R}^{N}} E\left(|\psi|^{2}\right)|\psi|^{2} d \mathbf{x} .
$$

So, for $p<2$ we have that $\tilde{h}_{\psi}\left(\lambda_{0}\right)>0$, that $\lambda_{0}<1$, and also that $h_{\psi}(\lambda)<0$ for $\lambda>1$.

Assume now that $p>2$ and $a>0$. In this case, we have that there is a unique $\lambda_{0}>0$ given by $(28)$. If we had $\lambda_{0}=1$, then we conclude that

$$
\frac{\|\psi\|_{p+2}^{p+2}}{\int_{\mathbb{R}^{N}} E\left(|\psi|^{2}\right)|\psi|^{2} d \mathbf{x}}=\frac{b(p+2)}{2 p a}, \quad \frac{\|\psi\|_{2}^{2}}{\int_{\mathbb{R}^{N}} E\left(|\psi|^{2}\right)|\psi|^{2} d \mathbf{x}}=\frac{b(p-2)}{p c}
$$

On the other hand, we also have for $p>2$ that $\int_{\mathbb{R}^{N}} E\left(|\psi|^{2}\right)|\psi|^{2} d \mathbf{x} \leq\|\psi\|_{4}^{4}=\left(\int_{|\psi| \leq 1}+\int_{|\psi| \geq 1}\right)|\psi(z)|^{4} d \mathbf{x} \leq$ $\|\psi\|_{2}^{2}+\|\psi\|_{p+2}^{p+2}$. which implies that

$$
1 \leq \frac{b(p+2)}{2 p a}+\frac{b(p-2)}{2 p c} \Leftrightarrow a(2 c p-(p-2) b)<b c(p+2) .
$$

If we assume for example that $c>b$, and choose $a>0$ such that

$$
a \geq \frac{b c(p+2)}{2 c p-(p-2) b},
$$

then we conclude that $\lambda_{0} \neq 1$. So, as in the first case, under the assumption that $a>0$ satisfies $(29)$, we have necessarily that $\lambda_{0}>1$, and so, we see directly that $h_{\psi}(\lambda)<0$ for $\lambda>1$, but close to $1^{+}$. Finally, the case $p=2$ and $a<b$ follows trivially as in the first case.

Now, we verify the condition (G5) only for $N=3$ (the case $N=2$ does not require this argument) for the operator $G_{5}(u)=\frac{b}{4} E\left(|u|^{2}\right)|u|^{2}$. Let $\psi, \phi \in H^{1}\left(\mathbb{R}^{3}\right)$ be such that $\operatorname{supp}(\psi) \cap \operatorname{supp}(\phi)=\emptyset$, then we have that

$$
\begin{aligned}
\int_{\mathbb{R}^{N}} G_{5}(\psi+\phi) d \mathbf{x} & =\int_{\mathbb{R}^{N}} E\left(|\psi+\phi|^{2}\right)|\psi+\phi|^{2} d \mathbf{x} \\
& =\int_{\mathbb{R}^{N}} E\left(|\psi|^{2}\right)|\psi|^{2} d \mathbf{x}+\int_{\mathbb{R}^{N}} E\left(|\phi|^{2}\right)|\phi|^{2} d \mathbf{x}+\int_{\mathbb{R}^{N}} 2 E\left(|\psi|^{2}\right)|\phi|^{2} d \mathbf{x} \\
& =\int_{\mathbb{R}^{N}} G_{5}(\psi) d \mathbf{x}+\int_{\mathbb{R}^{N}} G_{5}(\phi) d \mathbf{x}+\int_{\mathbb{R}^{N}} G_{0}(\psi, \phi) d \mathbf{x},
\end{aligned}
$$

where $G_{0}(\psi, \phi)=2 E\left(|\psi|^{2}\right)|\phi|^{2}$. Due to the nature of the operator $E$, we have that

$$
\int_{\mathbb{R}^{N}} G_{0}(\psi, \phi) d \mathbf{x}=\int_{\mathbb{R}^{N}} G_{0}(\phi, \psi) d \mathbf{x}=\int_{\text {supp } \phi} G_{0}(\psi, \phi) d \mathbf{x} .
$$




\subsection{Benney-Roskes/Zakharov-Rubenchik system}

The Benney-Roskes/Zakharov-Rubenchik system that describes the interaction of high-frequency and low-frequency waves in plasmas and magnetohydrodynamics given by

$$
\left\{\begin{aligned}
i \partial_{t} \psi+\epsilon \partial_{z}^{2} \psi & =-\sigma_{1} \Delta_{\perp} \psi+\left(\sigma|\psi|^{2}+W\left(\rho+D \partial_{z} \varphi\right)\right) \psi \\
\partial_{t} \rho+\sigma_{2} \partial_{z} \rho & =-\Delta_{\perp} \varphi-\partial_{z}^{2} \varphi-D \partial_{z}\left(|\psi|^{2}\right) \\
\partial_{t} \varphi+\sigma_{2} \partial_{z} \varphi & =-\frac{1}{M^{2}} \rho-|\psi|^{2}
\end{aligned}\right.
$$

We refer to [14, 1, 8] for more details on the physical background of this system. If we look for ground state solutions for the Benney-Roskes/Zakharov-Rubenchik system of the form

$$
\psi(\mathbf{x}, t)=e^{i c t} u(\mathbf{x}), \quad \rho(\mathbf{x}, t)=v(\mathbf{x}), \quad \varphi(\mathbf{x}, t)=w(\mathbf{x}),
$$

where $c>0$ and $(u, v, w) \in H^{1}\left(\mathbb{R}^{N}\right) \times L^{2}\left(\mathbb{R}^{N}\right) \times H^{1}\left(\mathbb{R}^{N}\right)$, then $u$ must satisfy the following problem

$$
\left\{\begin{array}{l}
-c u+\epsilon \partial_{z}^{2} u+\sigma_{1} \Delta_{\perp} u=\left(\sigma-M^{2} W\right)|u|^{2} u-W\left(D-M^{2} \sigma_{2}\right)^{2} E\left(|u|^{2}\right) u \\
u \in H^{1}\left(\mathbb{R}^{N}\right) \backslash\{0\}
\end{array}\right.
$$

where $v$ and $w$ are given by

$$
v=-M^{2} \sigma_{2} \partial_{z} w-M^{2}|u|^{2}, \quad \partial_{z} w=\left(M^{2} \sigma_{2}-D\right) E\left(|u|^{2}\right)
$$

and the operator $E$ is defined by its Fourier symbol as

$$
\widehat{E(u)}(\xi)=\Gamma_{2}(\xi) \widehat{u}(\xi), \quad \Gamma_{2}(\xi):=\frac{\xi_{3}^{2}}{\left(\xi_{1}^{2}+\xi_{2}^{2}+\left(1-M^{2}\right) \xi_{3}^{2}\right)},
$$

in the case $0<M<1$. In this case, we have that

$$
g_{5}(u)=\left(\sigma-W M^{2}\right)|u|^{2} u-W\left(M^{2} \sigma_{2}-D\right)^{2} E\left(|u|^{2}\right) u,
$$

with

$$
G_{5}(u)=\left(\sigma-W M^{2}\right)|u|^{4}-W\left(M^{2} \sigma_{2}-D\right)^{2} E\left(|u|^{2}\right) u^{2} .
$$

The condition (G1)-(G5) are verified by adapting previous case (see also the work by J. Quintero and J. Cordero [5]).

\subsection{A generalized Benney-Roskes/Zakharov-Rubenchik system}

As established by J. Quintero in [12]-13], the generalized nonlinear type Schrödinger equation,

$$
i \partial_{t} \psi+\epsilon \partial_{z}^{2} \psi+\sigma_{1} \Delta_{\perp} \psi=\sigma|\psi|^{p} \psi-M^{2} W|\psi|^{2} \psi-W\left(D-M^{2} \sigma_{2}\right)^{2} E\left(|\psi|^{2}\right) \psi
$$

in $R^{2}$ and $\mathbb{R}^{3}$ comes from the generalized Zakharov-Rubenchik system (or the Benney-Roses system) given by

$$
\left\{\begin{aligned}
i \partial_{t} \psi+\epsilon \partial_{z}^{2} \psi & =-\sigma_{1} \Delta_{\perp} \psi+\left(\sigma|\psi|^{p}+W\left(\rho+D \partial_{z} \varphi\right)\right) \psi \\
\partial_{t} \rho+\sigma_{2} \partial_{z} \rho & =-\Delta_{\perp} \varphi-\partial_{z}^{2} \varphi-D \partial_{z}\left(|\psi|^{2}\right) \\
\partial_{t} \varphi+\sigma_{2} \partial_{z} \varphi & =-\frac{1}{M^{2}} \rho-|\psi|^{2}
\end{aligned}\right.
$$

If we look for a ground state solutions

$$
\psi(\mathbf{x}, t)=e^{i c t} u(\mathbf{x}), \quad \rho(\mathbf{x}, t)=v(\mathbf{x}), \quad \varphi(\mathbf{x}, t)=w(\mathbf{x}),
$$


for the nonlinear type Schrödinger equation or the Benney-Roskes/Zakharov-Rubenchik system with $c>0,(u, v) \in$ $H^{1}\left(\mathbb{R}^{N}\right) \times L^{2}\left(\mathbb{R}^{N}\right)$ and $w_{z} \in L^{2}\left(\mathbb{R}^{N}\right)$, then $u$ must satisfy the following problem

$$
\left\{\begin{array}{l}
-c u+\epsilon \partial_{z}^{2} u+\sigma_{1} \Delta_{\perp} u=\sigma|u|^{p} u-M^{2} W|u|^{2} u-W\left(D-M^{2} \sigma_{2}\right)^{2} E\left(|u|^{2}\right) u \\
u \in H^{1}\left(\mathbb{R}^{N}\right) \backslash\{0\}
\end{array}\right.
$$

where $v$ and $w$ are given by

$$
v=-M^{2} \sigma_{2} \partial_{z} w-M^{2}|u|^{2}, \quad \partial_{z} w=\left(M^{2} \sigma_{2}-D\right) E\left(|u|^{2}\right) .
$$

and the operator $E$ is defined by its Fourier symbol as

$$
\widehat{E(u)}(\xi)=\Gamma_{3}(\xi) \widehat{u}(\xi), \quad \Gamma_{3}(\xi):=\frac{\xi_{3}^{2}}{\left(\xi_{1}^{2}+\xi_{2}^{2}+\left(1-M^{2}\right) \xi_{3}^{2}\right)},
$$

in the case $0<M<1$. In this case, we have that

$$
g_{6}(u)=\sigma|u|^{p} u-W M^{2}|u|^{2} u-W\left(M^{2} \sigma_{2}-D\right)^{2} E\left(|u|^{2}\right) u
$$

with

$$
G_{6}(u)=\sigma|u|^{p+2}-W M^{2}|u|^{4}-W\left(M^{2} \sigma_{2}-D\right)^{2} E\left(|u|^{2}\right) u^{2} .
$$

In this case, we see that existence of travelling waves follows by imposing the same restrictions on the parameters $a$ and $p$ stated by Rolci Cipolatti in [4] for the Davey-Stewardson system (case 3 ) given by,

$$
\begin{aligned}
& a_{0}(p)= \begin{cases}\infty, & \text { for } p<2 \\
b, & \text { for } p=2 \\
\left(\frac{p-2}{c(p+2)}\right)^{\frac{p}{p-2}}\left(\frac{(p+2) b}{2 p}\right)^{\frac{p}{2}}, & \text { for } p>2 .\end{cases} \\
& a_{1}(p)=\frac{b c(p+2)}{2 c p-(p-2) b}, \quad c>b, \quad p>2 .
\end{aligned}
$$

We also define the sets

$$
\mathcal{A}_{c, b}=\left\{(p, a): 0<p<p^{*}, \quad a<a_{0}(p)\right\}
$$

and

$$
\mathcal{B}_{c, b}=\left\{(p, a): 0<p<p^{*}, \quad a_{1}(p)<a<a_{0}(p)\right\}
$$

for $c>b$ and $p>2$ in the case $a_{1}(p)<a_{0}(p)$.

\section{Acknowledgments}

J. Quintero was supported by the Mathematics Department at Universidad del Valle (Colombia), under the research project CI 71231.

\section{References}

[1] Beney D. \& Roskes G. (1969). Wave Instability, Studies in Applied Math. 48, 455-472.

[2] Bahri A. \& Lions P. (1997). On the existence of a positive solution of semilinear elliptic equations in unbounded domains, Ann. Inst. H. Poincaré Anal. Non Lináire 14, 365-413. 
[3] Cazenave T. (1989). An introduction to nonlinear Schrodinger equations (Textos de Métodos Matemáticos Vol 22), UFRJ, Rio de Janeiro.

[4] Cipolatti R. (2020). On the existence of standing waves for a Davey-Stewartson system, Comm. Part. Diff. Eq. 17 (1992), 967-988.

[5] Cordero J. \& Quintero J. (2020). Instability of the Standing Waves for a Benney-Roskes/Zakharov-Rubenchik System and Blow-up for the Zakharov Equations, Discrete and Continuous Dynamical Systems Series B. 25 (4),1213-1240.

[6] Davey A. \& Stewartson K. (1974). On three-dimensional packets of surface waves. Proc. R. Soc. A. 338, $101-110$.

[7] Ghidaglia J. \& Saut J-C. (1996). Nonexistence of Travelling Wave Solutions to Nonelliptic Nonlinear Schriidinger Equations, Nonlinear Sci. 6, 139-145.

[8] Kuznetsov E. \& Zakharov V. (1984). Hamiltonian formalism for systems of hydrodynamics type, Mathematical Physics Review, Soviet Scientific Reviews, 4, 167-220.

[9] Lions P. (1984). The concentration-compactness principle in the calculus of variations. The locally compact case. Part I, Ann. Inst. H. Poincaré, Analyse non linéaire. 1, 109-145.

[10] Lions P. (1984). The concentration-compactness principle in the calculus of variations. The locally compact case. Part II, Ann. Inst. H. Poincaré, Analyse non linéaire. 1, 223-283.

[11] Ponce G. \& Saut J-C. (2005). Wellposedness for the Benney-Roskes/Zakharov-Rubenchik system, Discrete and Continuous Dynamical Systems. 13(3), 811-825.

[12] Quintero J. (2020). Stability and instability analysis for the Standing Waves for a generalized Zakharov-Rubenchik System. Preprint.

[13] Quintero J. (2020). Stability of Standing Waves for a generalized Zakharov-Rubenchik System. Preprint.

[14] Rubenchik A. \& Zakharov V. (1972). Nonlinear Interaction of High-Frequency and Low-Frequency Waves, Prikl. Mat. Techn. Phys. 5, 84-98.

[15] Tintarev K. (2006). A semilinear elliptic problem on unbounded domains with reverse penalty Nonlinear Analysis 64, 1496-1502 